TRANSACTIONS OF THE

AMERICAN MATHEMATICAL SOCIETY

Volume 362, Number 3, March 2010, Pages 1571-1597

S 0002-9947(09)04872-7

Article electronically published on October 20, 2009

\title{
DYADIC-LIKE MAXIMAL OPERATORS ON INTEGRABLE FUNCTIONS AND BELLMAN FUNCTIONS RELATED TO KOLMOGOROV'S INEQUALITY
}

\author{
ANTONIOS D. MELAS AND ELEFTHERIOS NIKOLIDAKIS
}

\begin{abstract}
For each $q<1$ we precisely evaluate the main Bellman functions associated with the behavior of dyadic maximal operators on $\mathbb{R}^{n}$ on integrable functions. Actually we do that in the more general setting of tree-like maximal operators. These are related to and refine the corresponding Kolmogorov inequality, which we show is actually sharp. For this we use the effective linearization introduced by the first author in 2005 for such maximal operators on an adequate set of functions.
\end{abstract}

\section{INTRODUCTION}

The dyadic maximal operator on $\mathbb{R}^{n}$ is defined by

$$
M_{d} \phi(x)=\sup \left\{\frac{1}{|Q|} \int_{Q}|\phi(u)| d u: x \in Q, Q \subseteq \mathbb{R}^{n} \text { is a dyadic cube }\right\}
$$

for every $\phi \in L_{\text {loc }}^{1}\left(\mathbb{R}^{n}\right)$, where the dyadic cubes are the cubes formed by the grids $2^{-N} \mathbb{Z}^{n}$ for $N=0,1,2, \ldots$

As is well known it satisfies the following weak type $(1,1)$ inequality:

$$
\left|\left\{x \in \mathbb{R}^{n}: M_{d} \phi(x)>\lambda\right\}\right| \leq \frac{1}{\lambda} \int_{\left\{M_{d} \phi>\lambda\right\}}|\phi(u)| d u
$$

for every $\phi \in L^{1}\left(\mathbb{R}^{n}\right)$ and every $\lambda>0$, from which follows, in view of Kolmogorov's inequality, the following $L^{q}$ inequality:

$$
\int_{E}\left|M_{d} \phi(u)\right|^{q} d u \leq \frac{1}{1-q}|E|^{1-q}\|\phi\|_{1}^{q}
$$

for every $q$ with $0<q<1$, every $\phi \in L^{1}\left(\mathbb{R}^{n}\right)$ and every measurable subset $E$ of $\mathbb{R}^{n}$ with finite measure.

It is easy to see that the weak type inequality (1.2) is best possible. We will show here that (1.3) is actually sharp.

An approach for studying such maximal operators is the introduction of the so-called Bellman functions (see [5]) related to them which reflect certain deeper

Received by the editors August 21, 2007 and, in revised form, April 7, 2008.

2000 Mathematics Subject Classification. Primary 42B25.

Key words and phrases. Bellman, dyadic, maximal.

The authors were supported in part by the European Social Fund and National Resources(EPEAK II) Pythagoras II. 
properties of them by localizing. Such functions related to the $L^{p}$ for $p>1$ have been precisely evaluated in [4].

The Bellman function related to the present situation is defined for any $0<q<1$ as follows:

$$
\begin{gathered}
\mathcal{B}_{q}(f, h, L, k)=\sup \left\{\frac{1}{|Q|} \int_{E}\left(M_{d} \phi\right)^{q}: \operatorname{Av}_{Q}(\phi)=f, \operatorname{Av}_{Q}\left(\phi^{q}\right)=h,\right. \\
\left.\sup _{Q^{\prime}: Q \subseteq Q^{\prime}} \operatorname{Av}_{Q^{\prime}}(\phi)=L, E \subseteq Q \text { measurable with }|E|=k\right\},
\end{gathered}
$$

where $Q$ is a fixed dyadic cube, $Q^{\prime}$ runs over all dyadic cubes containing $Q, \phi$ is nonnegative in $L^{1}(Q)$ and the variables $f, h, L$ and satisfy $0 \leq f \leq L, h \leq f^{q}$ and $0 \leq k \leq 1 . \mathcal{B}_{q}$ is independent of the choice of $Q$ (so we may take $Q=[0,1]^{n}$ ).

There are several other problems in Harmonic Analysis where Bellman functions naturally arise. Such problems (including the dyadic Carleson imbedding and weighted inequalities) are described in [7] (see also [5, [6]) and also connections to Stochastic Optimal Control are provided, from which it follows that the corresponding Bellman functions satisfy certain nonlinear second-order PDEs.

The exact computation of a Bellman function is a difficult task which is connected with the deeper structure of the corresponding Harmonic Analysis problem. Thus far several Bellman functions have been computed (see [1, 2], 4, 8], 10, [12, [13, 14]). Recently L. Slavin and A. Stokolos [9] in some cases linked the Bellman function computation to solving certain PDEs of the Monge-Ampère type, and in this way they obtained an alternative proof of the results in 4] for the Bellman functions related to the dyadic maximal operator. Also in [14] using the Monge-Ampère equation approach a more general Bellman function than the one related to the dyadic Carleson Imbedding Theorem has been precisely evaluated, thus generalizing the corresponding result in [4.

Here we will compute the Bellman function defined by (1.4). The computation of the above Bellman functions will provide refinements of the local $L^{q}$ inequality (1.3), which as we will show in the process is sharp. Our approach will not use the Bellman PDE but will rely on a deeper study of the combinatorial structure of these maximal operators in the same way as in [4. However the analysis as well as the results will be in certain aspects different due to the concavity of the corresponding $q$ power function and in this sense the results of this paper can be considered as complementing those in 44. Actually we will define and compute such functions on the more general situation of tree-like structures on probability spaces and as in [4] show that they are always the same.

As in [4] let $(X, \mu)$ be a nonatomic probability space and let $\mathcal{T}$ be a family of measurable subsets of $X$ that has a tree-like structure similar to the one in the dyadic case (the precise definition will be given in the next section). Then we can define the maximal operator associated to $\mathcal{T}$ as follows:

$$
M_{\mathcal{T}} \phi(x)=\sup \left\{\frac{1}{\mu(I)} \int_{I}|\phi| d \mu: x \in I \in \mathcal{T}\right\}
$$

for every $\phi \in L^{1}(X, \mu)$. 
The above maximal operator is related to the theory of martingales and satisfies essentially the same inequalities as $M_{d}$. Now we define the corresponding Bellman function as

$$
\begin{gathered}
\mathcal{B}_{q}^{\mathcal{T}}(f, h, L, k)=\sup \left\{\int_{E}\left(\max \left(M_{\mathcal{T}} \phi, L\right)\right)^{q} d \mu: \phi \geq 0, \phi \in L^{1}(X, \mu),\right. \\
\left.\int_{X} \phi d \mu=f, \int_{X} \phi^{q} d \mu=h, E \subseteq X \text { measurable with }|E|=k\right\},
\end{gathered}
$$

where again the variables satisfy $L \geq f>0,0<h \leq f^{q}$ and $0 \leq k \leq 1$. It is easy to see that when $X=[0,1]^{n}, \mu$ is the Lebesgue measure and $\mathcal{T}$ is the family of dyadic cubes contained in $X$, the above function becomes the Bellman function $\mathcal{B}_{q}$ defined by (1.4) since we can define $\phi$ on $[0,2]^{n} \backslash X$ (and set it equal to 0 outside $\left.[0,2]^{n}\right)$ to make $\sup _{Q^{\prime}: X \subseteq Q^{\prime}} \operatorname{Av}_{Q^{\prime}}(\phi)=\operatorname{Av}_{[0,2]^{n}}(\phi)=L$. Also in the case where $X, \mathcal{T}$ are part of a larger tree-like structure, $\mathcal{B}_{q}^{\mathcal{T}}$ can be defined in a way analogous to (1.4).

To state our main result we consider for any $0<q<1$ the function

$$
H_{q}(z)=(1-q) z^{q}+q z^{q-1}
$$

defined for $z>0$. Since $H_{q}^{\prime}(z)=(1-q) q z^{q-2}(z-1)$ it is easy to see that $H_{q}$ is strictly increasing on the interval $[1,+\infty)$, strictly decreasing on $(0,1]$ and it maps each of them onto $[1,+\infty)$. We now define $\omega_{q}:[1,+\infty) \rightarrow[1,+\infty)$ by

$$
\omega_{q}(z)=\left(H_{q}^{-1}(z)\right)^{q}
$$

where $H_{q}^{-1}$ denotes the inverse function of the restriction of $H_{q}$ on $[1,+\infty)$. Clearly $\omega_{q}$ is strictly increasing, maps $[1,+\infty)$ onto $[1,+\infty)$, and can be defined by the equation

$$
(1-q) \omega_{q}(z)+q \omega_{q}(z)^{1-\frac{1}{q}}=z .
$$

For example for $q=1 / 2$ we have $\omega_{1 / 2}(z)=z+\sqrt{z^{2}-1}$.

Next as we shall show in Lemma 3 for any $\lambda>1$ and $0<k<1$ the equation

$$
H_{q}\left(\frac{x(1-k)}{1-k x}\right)=\lambda H_{q}(x)
$$

has a unique solution $x=x(\lambda, k)$ in the interval $\left(1, \frac{1}{k}\right)$. Then we have the following.

Theorem 1. For any nonatomic probability space $(X, \mu)$, any tree-like family $\mathcal{T}$ and any $0<q<1$ the corresponding Bellman function is given by

$$
\mathcal{B}_{q}^{\mathcal{T}}(f, h, L, k)=\left\{\begin{array}{l}
h \omega_{q}\left(\frac{f^{q}}{h} H_{q}\left(\frac{L}{f}\right)\right)-L^{q}(1-k), k_{0}(f, h, L) \leq k \leq 1, \\
h \omega_{q}\left(\frac{f^{q}}{h} H_{q}\left(x\left(\frac{f^{q}}{h}, k\right)\right)\right)-f^{q} x\left(\frac{f^{q}}{h}, k\right)^{q}(1-k), 0<k \leq k_{0}(f, h, L),
\end{array}\right.
$$


where

$$
k_{0}(f, h, L)=\frac{f \omega_{q}\left(\frac{f^{q}}{h} H_{q}\left(\frac{L}{f}\right)\right)^{1 / q}-L}{L\left(\omega_{q}\left(\frac{f^{q}}{h} H_{q}\left(\frac{L}{f}\right)\right)^{1 / q}-1\right)}
$$

is the unique in $\left(0, \frac{f}{L}\right)$ solution of the equation $H_{q}\left(\frac{L(1-k)}{f-L k}\right)=\frac{f^{q}}{h} H_{q}\left(\frac{L}{f}\right)$.

Thus the Bellman functions are the same for any such $X, \mathcal{T}$ and depend only on the underlying tree-like structure. Note that since $f^{q}>h L>f$ and

$$
\omega_{q}\left(\frac{f^{q}}{h} H_{q}\left(\frac{L}{f}\right)\right)^{1 / q}>\omega_{q}\left(H_{q}\left(\frac{L}{f}\right)\right)^{1 / q}=\frac{L}{f}
$$

the expression in (1.12) is indeed in $\left(0, \frac{f}{L}\right)$.

Considering the various special cases $k=1, L=f \quad$ (where for example in the case $L=f$ the function $\mathcal{B}_{q}^{\mathcal{T}}(f, h, f, k)$ becomes the supremum of $\int_{E}\left(M_{\mathcal{T}} \phi\right)^{q} d \mu$ when $\phi \geq 0, \int_{X} \phi d \mu=f, \int_{X} \phi^{q} d \mu=h$ and $E \subseteq X$ is measurable with $|E|=k$ ) we get the following:

Corollary 1. For any nonatomic probability space $(X, \mu)$ and any tree-like family $\mathcal{T}$, any $\phi \geq 0$ measurable with $\int_{X} \phi d \mu=f \leq L, \int_{X} \phi^{q} d \mu=h$ and any measurable $E \subseteq X$ with $\mu(E)=k \in[0,1]$, we have the following sharp inequalities (where sharpness means that their right-hand side is exactly the supremum over all $\phi, E$ involved with the corresponding properties):

$$
\begin{aligned}
& \int_{X}\left(M_{\mathcal{T}} \phi\right)^{q} d \mu \leq h \omega_{q}\left(\frac{f^{q}}{h}\right), \\
& \int_{X}\left(\max \left(M_{\mathcal{T}} \phi, L\right)\right)^{q} d \mu \leq h \omega_{q}\left(\frac{(1-q) L^{q}+q L^{q-1} f}{h}\right), \\
& \int_{E}\left(M_{\mathcal{T}} \phi\right)^{q} d \mu \leq h \omega_{q}\left(\frac{f^{q}}{h} H_{q}\left(x\left(\frac{f^{q}}{h}, k\right)\right)\right)-f^{q} x\left(\frac{f^{q}}{h}, k\right)^{q}(1-k) .
\end{aligned}
$$

Actually the above inequalities will be the basic steps in proving Theorem 1, starting with (1.13). Note that in [4 the Bellman function analogous to (1.14) for $p>1$ was given by a double formula and was not $C^{\infty}$, a relation analogous to (1.14) holding only for $L$ smaller than $\frac{p}{p-1} f$. However in the present situation this function is given by a single formula and is smooth.

In view of this corollary the double expression in (1.11) of Theorem 1 can be explained as follows. If $k$ is small enough, that is, if $k<k_{0}(f, h, L)$, then the supremum $\int_{E}\left(\max \left(M_{\mathcal{T}} \phi, L\right)\right)^{q} d \mu$ is taken ignoring $L$, that is when $M_{\mathcal{T}} \phi>L$ on $E$, whereas when $k \geq k_{0}(f, h, L)$, then the supremum is taken when $M_{\mathcal{T}} \phi \leq L$ on the complement of $E$. The reason for this can be inferred from the proof of Theorem 1. The number $k_{0}(f, h, L)$ as we shall see in section 6 is close to $\frac{f}{L}$ when $h$ is small (compared to $f^{q}$ ).

We will also show that every extremal sequence for (1.13) exhibits a certain self-similar homogeneous behavior (which in case $h<f^{q}$ shows that there are no 
extremal functions for it). This is contained in the following (and its proof will use the independence of the Bellman functions from $X, \mathcal{T})$.

Proposition 1. If $\left(\phi_{m}\right)$ is a sequence of nonnegative functions in $L^{1}(X, \mu)$ such that $\int_{X} \phi_{m} d \mu=f, \int_{X} \phi_{m}^{q} d \mu=h$ for all $m$ and

$$
\lim _{m \rightarrow \infty} \int_{X}\left(M_{\mathcal{T}} \phi_{m}\right)^{q} d \mu=h \omega_{q}\left(\frac{f^{q}}{h}\right)
$$

then for every $I \in \mathcal{T}$ we have:

$$
\begin{gathered}
\lim _{m \rightarrow \infty} \frac{1}{\mu(I)} \int_{I} \phi_{m} d \mu=f, \lim _{m \rightarrow \infty} \frac{1}{\mu(I)} \int_{I} \phi_{m}^{q} d \mu=h \text { and } \\
\lim _{m \rightarrow \infty} \frac{1}{\mu(I)} \int_{I}\left(M_{\mathcal{T}} \phi_{m}\right)^{q} d \mu=h \omega_{q}\left(\frac{f^{q}}{h}\right) .
\end{gathered}
$$

That is, an extremal sequence for $\mathcal{B}_{q}^{\mathcal{T}}(f, h, f, 1)$ is also extremal, for the same values of the variables, when localized on any element of the tree $\mathcal{T}$.

In the special case where $q=1 / 2$ the expressions in (1.13) and (1.14) can be explicitly computed and they yield the following sharp inequalities:

$$
\begin{aligned}
& \int_{X} \sqrt{M_{\mathcal{T}} \phi} d \mu \leq \sqrt{f}+\sqrt{f-h^{2}}<2 f \\
& \int_{X} \sqrt{\max \left(M_{\mathcal{T}} \phi, L\right)} d \mu \leq \frac{1}{2}\left(\sqrt{L}+\frac{f}{\sqrt{L}}\right)+\sqrt{\frac{1}{4}\left(\sqrt{L}+\frac{f}{\sqrt{L}}\right)^{2}-h^{2}} .
\end{aligned}
$$

For the general $q$ in order to compare the above inequalities with what Kolmogorov's inequality would give we will obtain appropriate estimates for the above expressions. Then, after scaling these expressions, produce improvements, in view of the Bellman function (1.4), of Kolmogorov's inequality on the dyadic maximal operator on $\mathbb{R}^{n}$. This will be done in section 6 .

This paper is organized as follows. In section 2 we collect a number of technical lemmas needed throughout this paper. In section 3 we prove (1.13) (and its sharpness) using the linearization for the maximal operators introduced in [4] and also prove certain improvements of it under more stringent conditions on $\phi$. In section 4 we compute successively the specializations of the Bellman function (1.6) reaching the proof of our Theorem 1 and in section 5 we prove Proposition 1. In section 6 we derive certain approximate expressions for the various Bellman functions and study how they behave under scaling, thus obtaining improved versions of Kolmogorov's inequality on maximal operators which show its sharpness and explain how our main Theorem 1 is related to it.

\section{Some teChNicAl LEMMAS}

In this section we collect certain technical results that will be used throughout this paper. The first concerns the functions $H_{q}$ and $\omega_{q}$ defined in the introduction.

Lemma 1. Let $0<q<1$ be fixed. Then

(i) The function $\omega_{q}:[1,+\infty) \rightarrow[1,+\infty)$ is strictly increasing, strictly concave and for $x>1$ satisfies

$$
\frac{d}{d x} \omega_{q}(x)=\frac{1}{1-q} \frac{\omega_{q}(x)^{1 / q}}{\omega_{q}(x)^{1 / q}-1} .
$$


(ii) We have

$$
1<\frac{\omega_{q}(x)}{x}<\frac{1}{1-q}
$$

for every $x>1$ and

$$
\lim _{x \rightarrow+\infty} \frac{\omega_{q}(x)}{x}=\frac{1}{1-q} .
$$

(iii) The function $U(x)=\frac{\omega_{q}(x)}{x}$ is strictly increasing on $[1,+\infty)$.

(iv) We have

$$
\frac{x-q x^{1-\frac{1}{q}}}{1-q}<\omega_{q}(x)<\frac{x-q(1-q)^{\frac{1}{q}-1} x^{1-\frac{1}{q}}}{1-q} .
$$

Proof. (2.1) is an easy computation, and then the concavity follows since (2.1) and $\omega_{q}(x)>1$ for $x>1$ imply that $\omega_{q}^{\prime}(x)$ is strictly decreasing. Moreover (2.2) follows since $\omega_{q}(x)>x=(1-q) \omega_{q}(x)+q \omega_{q}(x)^{1-\frac{1}{q}}>(1-q) \omega_{q}(x)$ and (2.3) follows since $\frac{\omega_{q}(x)}{x}$ is bounded and $\omega_{q}(x)^{-\frac{1}{q}}$ tends to 0 as $x \rightarrow+\infty$. Now (iii) follows since

$$
\begin{aligned}
U^{\prime}(x) & =\frac{1}{x}\left(\frac{1}{1-q} \frac{\omega_{q}(x)^{1 / q}}{\omega_{q}(x)^{1 / q}-1}-\frac{\omega_{q}(x)}{(1-q) \omega_{q}(x)+q \omega_{q}(x)^{1-\frac{1}{q}}}\right) \\
& =\frac{q \omega_{q}(x)}{(1-q) x^{2}\left(\omega_{q}(x)^{1 / q}-1\right)}>0
\end{aligned}
$$

for $x>1$ and then (2.4) follows from (iii) and (2.2) since

$$
x^{\frac{1}{q}-1}\left(x-(1-q) \omega_{q}(x)\right)=q\left(\frac{\omega_{q}(x)}{x}\right)^{1-\frac{1}{q}} .
$$

Lemma 2. Let $0<q<1$ and $\lambda>1$. Then for every $\alpha$ with $0<\alpha<1$ the equation

$$
z^{q}-(1-\alpha)^{1-q}(z-\alpha)^{q}=\lambda \alpha
$$

has a unique solution $z=z_{q}(\alpha, \lambda)$ in $(1,+\infty)$ and moreover $\alpha \rightarrow z_{q}(\alpha, \lambda)$ is strictly decreasing with

$$
z_{q}(\alpha, \lambda)<\frac{\alpha}{1-(1-\alpha)^{1-q}} \lambda \text { and } \lim _{a \rightarrow 0^{+}} z_{q}(\alpha, \lambda)^{q}=\omega_{q}(\lambda) .
$$

Proof. Let $F(\alpha, z)=\frac{z^{q}-(1-\alpha)^{1-q}(z-\alpha)^{q}}{\alpha}$. Then $F_{\alpha}(1)=1$ and

$$
\frac{F(\alpha, z)}{z^{q}}=\frac{1}{\alpha}\left(1-(1-\alpha)^{1-q}\left(1-\frac{\alpha}{z}\right)^{q}\right)>\frac{1}{\alpha}\left(1-(1-\alpha)^{1-q}\right)>1-q
$$

whenever $z>1$; thus $\lim _{z \rightarrow+\infty} F(\alpha, z)=+\infty$. On the other hand,

$$
\frac{\partial F(\alpha, z)}{\partial z}=\frac{q}{\alpha}\left(\frac{1}{z^{1-q}}-\left(\frac{1-\alpha}{z-\alpha}\right)^{1-q}\right)>0
$$

for every $z>1$. Hence (2.5) has a unique solution $z=z_{q}(\alpha, \lambda)$ in $(1,+\infty)$. Moreover by (2.7) the first inequality in (2.6) easily follows, which also implies that $z_{q}(\alpha, \lambda)$ is bounded as $\alpha \rightarrow 0^{+}$. But then whenever $\alpha_{m} \rightarrow 0^{+}$and $z_{m}=$ $z_{q}\left(\alpha_{m}, \lambda\right) \rightarrow z^{*}$ we have

$$
\lambda=\frac{z_{m}^{q}-\left(z_{m}-\alpha_{m}\right)^{q}}{\alpha_{m}}+\left(z_{m}-\alpha_{m}\right)^{q} \frac{1-\left(1-\alpha_{m}\right)^{1-q}}{\alpha_{m}} \rightarrow H_{q}\left(z^{*}\right)
$$


and this proves the other part of (2.6). To show that $\alpha \rightarrow z_{q}(\alpha, \lambda)$ is strictly decreasing just note that

$$
\frac{\partial F(\alpha, z)}{\partial \alpha}=-\frac{1}{\alpha^{2}}\left(z^{q}-(1-q \alpha)\left(\frac{z-\alpha}{1-\alpha}\right)^{q}-q\left(\frac{z-\alpha}{1-\alpha}\right)^{q-1}\right)
$$

and with $w=\frac{1-\alpha}{z-\alpha} \in(0,1)$ use the inequalities $(\alpha w+1-\alpha)^{q}=(1-\alpha(1-w))^{q}<$ $1-q a(1-w)<1-q a+q w$ to get $\frac{\partial F(\alpha, z)}{\partial \alpha}>0$.

For the next lemma (and with $0<q<1$ ) we consider the function

$$
\sigma_{q}(k, x)=\frac{H_{q}\left(\frac{x(1-k)}{1-k x}\right)}{H_{q}(x)}
$$

defined for all $k, x$ such that $0<k<1$ and $0<x<\frac{1}{k}$. A straightforward computation shows that

$$
\sigma_{q}(k, x)=\frac{(1-q) x+q-k x}{(1-k)^{1-q}(1-k x)^{q}((1-q) x+q)}
$$

and

$$
\begin{gathered}
\frac{\partial \sigma_{q}}{\partial k}(k, x)=D_{q}(k, x)(x-1)^{2} \\
\frac{\partial \sigma_{q}}{\partial x}(k, x)=D_{q}(k, x)(x-1) k(1-k) \frac{(1-q) x+q+1-k x}{(1-q) x+q}
\end{gathered}
$$

where

$$
D_{q}(k, x)=\frac{(1-q) q(1-k)^{q-2} x^{q-1}}{H_{q}(x)(1-k x)^{q+1}}>0
$$

on the domain of definition of $\sigma_{q}(k, x)$. We then have the following.

Lemma 3. (i) For any fixed $\lambda>1$ the equation

$$
H_{q}\left(\frac{x(1-k)}{1-k x}\right)=\lambda H_{q}(x)
$$

has a unique solution $x=x(\lambda, k)=x_{\lambda}(k)$ in the interval $\left(1, \frac{1}{k}\right)$, and it has a solution in the interval $(0,1)$ if and only if $\lambda<(1-k)^{q-1}$, in which case this is also unique. Moreover the function $x_{\lambda}$ on $(0,1)$ is smooth strictly decreasing and it satisfies $\lim _{k \rightarrow 1^{-}} x_{\lambda}(k)=1, \lim _{k \rightarrow 0^{+}} x_{\lambda}(k)=+\infty$ and

$$
x_{\lambda}^{\prime}(k)=-\frac{\left(x_{\lambda}(k)-1\right)\left((1-q) x_{\lambda}(k)+q\right)}{k(1-k)\left((1-q) x_{\lambda}(k)+q+1-k x_{\lambda}(k)\right)} .
$$

(ii) If $\mu \geq 0$ and we define

$$
R_{q, \mu}(k, x)=\left(\frac{x(1-k)}{1-k x}\right)^{q} \frac{1}{\sigma_{q}(k, x)}+\left(\mu^{q}-x^{q}\right)(1-k)
$$

on $W=\left\{(k, x): 0<k<1\right.$ and $\left.1<x<\frac{1}{k}\right\}$, then

$$
\frac{d}{d k} R_{q, \mu}\left(k, x_{\lambda}(k)\right)=x_{\lambda}(k)^{q}-\mu^{q} .
$$


(iii) If $\mu>1$ and $\xi$ is in $(0,1]$ the maximum value of $R_{q, \mu}$ on the set $\{(k, x) \in W$ : $0<k \leq \xi$ and $\left.\sigma_{q}(k, x)=\lambda\right\}$ is equal to $\frac{1}{\lambda} \omega_{q}\left(\lambda H_{q}(\mu)\right)$ if $\xi \geq k_{0}(\lambda, \mu)$ and it is equal to $R_{q, \mu}\left(\xi, x_{\lambda}(\xi)\right)$ if $\xi<k_{0}(\lambda, \mu)$, where

$$
k_{0}(\lambda, \mu)=\frac{\omega_{q}\left(\lambda H_{q}(\mu)\right)^{1 / q}-\mu}{\mu\left(\omega_{q}\left(\lambda H_{q}(\mu)\right)^{1 / q}-1\right)}
$$

is the unique in $\left(0, \frac{1}{\mu}\right)$ solution of the equation $\sigma_{q}\left(k_{0}, \mu\right)=\lambda$.

(iv) We have

$$
\frac{1}{k}\left(1-(1-k) \lambda^{-1 / q}\right)<x_{\lambda}(k)<\frac{1}{k}\left(1-\left(\frac{1-q}{1-q+k}\right)^{1 / q}(1-k) \lambda^{-1 / q}\right) .
$$

Proof. (i) The second equation in (2.12) implies $(x-1) \frac{\partial \sigma_{q}}{\partial x}>0$ if $x \neq 1$. Since, for any fixed $k, \lim _{x \rightarrow \frac{1}{k}^{-}} \sigma_{q}(x, k)=+\infty$ and by (2.11), $\lim _{x \rightarrow 0^{+}} \sigma_{q}(x, k)=(1-k)^{q-1}$ the first part of (i) follows. Now (2.15) follows from (2.12) and $\lim _{k \rightarrow 1^{-}} x_{\lambda}(k)=1$ follows since $1<x_{\lambda}(k)<\frac{1}{k}$. To prove that $\lim _{k \rightarrow 0^{+}} x_{\lambda}(k)=+\infty$ observe that if there existed $k_{m} \rightarrow 0^{+}$such that $x_{m}=x_{\lambda}\left(k_{m}\right) \rightarrow x^{*}<+\infty$, then $k_{m} x_{m} \rightarrow 0$ and so (2.12) would give $\lambda=\sigma_{q}\left(k_{m}, x_{m}\right) \rightarrow 1$, a contradiction.

(ii) The equality (2.17) follows from a straightforward but tedious computation using (2.15), (2.12) and the fact that $\sigma_{q}\left(k, x_{\lambda}(k)\right)=\lambda$ for all $k$ (so the term $1 / \sigma_{q}$ in (2.16) is not differentiated, being constant, but is replaced not by $\lambda$ but by the expression in (2.11) with $\left.x=x_{\lambda}(k)\right)$.

(iii) By (i) there exists a unique $k_{0}=k_{0}(q, \lambda, \mu)$ in $(0,1)$ such that $x_{\lambda}\left(k_{0}\right)=\mu$ and since $x_{\lambda}$ is strictly decreasing we have using (2.17) that $\frac{d}{d k} R_{q, \mu}\left(k, x_{\lambda}(k)\right)$ is $>0$ if $k<k_{0}$ and $<0$ if $k>k_{0}$; hence the maximum value of $R_{q, \mu}$ on the set $\left\{(k, x) \in W: 0<k \leq \xi\right.$ and $\left.\sigma_{q}(k, x)=\lambda\right\}$ is equal to $R_{q, \mu}\left(\xi, x_{\lambda}(\xi)\right)$ if $\xi<k_{0}$ and to $R_{q, \mu}\left(k_{0}, x_{\lambda}\left(k_{0}\right)\right)$ if $\xi \geq k_{0}$. However since $\mu=x_{\lambda}\left(k_{0}\right)<\frac{1}{k_{0}}$ we have $k_{0}<\frac{1}{\mu}$ and now $\sigma_{q}\left(k_{0}, \mu\right)=\sigma_{q}\left(k_{0}, x_{\lambda}\left(k_{0}\right)\right)=\lambda$ gives $\frac{\mu\left(1-k_{0}\right)}{1-k_{0} \mu}=H_{q}^{-1}\left(\lambda H_{q}(\mu)\right)$ from which the expression (2.18) follows. Moreover noting that

$$
\left(\frac{x_{\lambda}\left(k_{0}\right)\left(1-k_{0}\right)}{1-k_{0} x_{\lambda}\left(k_{0}\right)}\right)^{q}=\omega_{q}\left(H_{q}\left(\frac{x_{\lambda}\left(k_{0}\right)\left(1-k_{0}\right)}{1-k_{0} x_{\lambda}\left(k_{0}\right)}\right)\right)=\omega_{q}\left(\lambda H_{q}\left(x_{\lambda}\left(k_{0}\right)\right)\right)=\omega_{q}\left(\lambda H_{q}(\mu)\right)
$$

we get $R_{q, \mu}\left(k_{0}, x_{\lambda}\left(k_{0}\right)\right)=\frac{1}{\lambda} \omega_{q}\left(\lambda H_{q}(\mu)\right)$ and this proves (iii).

(iv) We write $x_{\lambda}(k)=\frac{1}{k}(1-(1-k) \theta)$, where $0<\theta<1$. Note that, in view of (2.11), $\theta$ satisfies

$$
\lambda \theta^{q}=\frac{(1-q)(1-\theta)+k \theta}{(1-q)(1-(1-k) \theta)+q k}=\rho_{k, \lambda}(\theta)
$$

and observe that $\frac{1-q}{1-q+k}=\rho_{k, \lambda}(0)<\rho_{k, \lambda}(y)<\rho_{k, \lambda}(1)=1$ for any $y \in(0,1)$. 
Now for the next lemma we fix real numbers $f, h$ and $k$ with $f, h>0, h<f^{q}$ and $0<k<1$ and we consider the functions

$$
l_{k}(B)=(1-k)^{1-q}(f-B)^{q}+k^{1-q} B^{q}
$$

defined for $0 \leq B \leq f$ and

$$
R_{k}(B)=\left\{\begin{array}{l}
\left(h-(1-k)^{1-q}(f-B)^{q}\right) \omega_{q}\left(\frac{k^{1-q} B^{q}}{h-(1-k)^{1-q}(f-B)^{q}}\right) \\
\text { if }(1-k)^{1-q}(f-B)^{q}<h \leq l_{k}(B), \\
\frac{k^{1-q} B^{q}}{1-q} \text { if } h \leq(1-k)^{1-q}(f-B)^{q}
\end{array}\right.
$$

defined for all $B \in[0, f]$ such that $l_{k}(B) \geq h$.

Noting that $l_{k}$ has an absolute maximum at $B=k f$ with $l_{k}(k f)=f^{q}>h$ and that it is monotone on each of the intervals $(0, k f)$ and $(k f, f)$ we conclude that either $l_{k}(f)<h$, i.e. $k^{1-q} f^{q}<h$, in which case the equation $l_{k}(B)=h$ has a unique solution in $(k f, f)$ and this is denoted by $\rho_{1}=\rho_{1}(f, h, k)$, or $l_{k}(f) \geq h$ in which case we set $\rho_{1}=\rho_{1}(f, h, k)=f$. Also either $l_{k}(0)<h$, i.e. $(1-k)^{1-q} f^{q}<h$, in which case the equation $l_{k}(B)=F$ has a unique solution in $(0, k f)$ and this is denoted by $\rho_{0}=\rho_{0}(f, h, k)$, or $h_{k}(0) \leq F$ in which case we set $\rho_{0}=\rho_{0}(f, h, k)=$ 0 . In all cases the domain of definition of $R_{k}$ is the interval $W_{k}=W_{k}(f, h)=$ $\left[\rho_{0}(f, h, k), \rho_{1}(f, h, k)\right]$. We now have the following.

Lemma 4. The maximum value of the function $R_{k}$ on $W_{k}$ is attained at the unique point $B^{*}=x_{\lambda}(k) k f>k f$, where $\lambda=\frac{f^{q}}{h}$ (see Lemma 3$)$, which also satisfies

$$
(1-k)^{1-q}\left(f-B^{*}\right)^{q}<h<l_{k}\left(B^{*}\right)
$$

and moreover we have

$$
\max _{W_{k}} R_{k}=h \omega_{q}\left(\frac{f^{q}}{h} H_{q}\left(x_{\lambda}(k)\right)\right)-(1-k) f^{q} x_{\lambda}(k)^{q} .
$$

Proof. Clearly $(1-k)^{1-q}(f-B)^{q}<h$ if and only if $\eta_{0}=\max \left(0, f-\frac{h^{\frac{1}{q}}}{(1-k)^{\frac{1}{q}-1}}\right)<$ $B \leq f, \eta_{0}<\rho_{1}(f, h, k)$ and $R_{k}(B)$ is strictly increasing in $W_{k} \cap\left(0, \eta_{0}\right]$ (if nonempty). Hence it suffices to find its maximum value on $W_{k}^{\prime}=W_{k} \cap\left[\eta_{0}, f\right]=\left[\max \left(\rho_{0}, \eta_{0}\right), \rho_{1}\right]$. In the interior of $W_{k}^{\prime}$ we have (using Lemma 1)

$$
R_{k}^{\prime}(B)=\frac{q}{1-q} \frac{\omega_{q}(Z(B))^{1 / q}}{\omega_{q}(Z(B))^{1 / q}-1}\left[\left(\frac{B}{k}\right)^{q-1}-\omega_{q}(Z(B))^{\frac{q-1}{q}}\left(\frac{f-B}{1-k}\right)^{q-1}\right],
$$

where

$$
Z(B)=\frac{k^{1-q} B^{q}}{h-(1-k)^{1-q}(f-B)^{q}}>1
$$

in the interior of $W_{k}^{\prime}$ and so $\omega_{q}(Z(B))>1$ there.

Now if $\rho_{1}=f$, then since $q<1$ we have $\lim _{B \rightarrow \rho_{1}^{-}} R_{k}^{\prime}(B)=-\infty$. Suppose $\rho_{1}<f$.

Then $\lim _{B \rightarrow \rho_{1}^{-}} Z(B)=1$ and

$$
\lim _{B \rightarrow \rho_{1}^{-}}\left(\left(\frac{B}{k}\right)^{q-1}-\omega_{q}(Z(B))^{\frac{q-1}{q}}\left(\frac{f-B}{1-k}\right)^{q-1}\right)=\left(\frac{\rho_{1}}{k}\right)^{q-1}-\left(\frac{f-\rho_{1}}{1-k}\right)^{q-1}<0
$$


since $\rho_{1}>k f$. Hence $\lim _{B \rightarrow \rho_{1}^{-}} R_{k}^{\prime}(B)=-\infty$ holds always. In an analogous way (and since $\rho_{0}<k f$ ) we have $\lim _{B \rightarrow \rho_{0}^{+}} R_{k}^{\prime}(B)=+\infty$ in the case where $\rho_{0}>\eta_{0}$. Moreover if $k f>\eta_{0}$, which happens if and only if $(1-k) f^{q}<h$, then since $Z(k f)>1$ and $q<1$ we get from (2.24) that $R_{k}^{\prime}(k f)>0$.

But by (2.24) we have $R_{k}^{\prime}(B)=0$ if and only if $\omega_{q}(Z(B))^{1 / q}=\frac{B(1-k)}{k(f-B)}$, which is equivalent to

$$
\begin{aligned}
H_{q}\left(\frac{B(1-k)}{k(f-B)}\right) & =(1-q)\left(\frac{B(1-k)}{k(f-B)}\right)^{q}+q\left(\frac{B(1-k)}{k(f-B)}\right)^{q-1} \\
& =\frac{k^{1-q} B^{q}}{h-(1-k)^{1-q}(f-B)^{q}}>1
\end{aligned}
$$

and provided that $h \neq(1-k)^{1-q}(f-B)^{q}$ this can been written as

$$
h H_{q}\left(\frac{B(1-k)}{k(f-B)}\right)=f^{q} H_{q}\left(\frac{B}{k f}\right) .
$$

Consider now the equation (2.26) on the larger interval $(0, f)$. Since $h<f^{q}$ the number $B=k f$ is not a solution of it and since $H_{q}(z)>1$ for all $z \neq 1$ we get $H_{q}\left(\frac{B(1-k)}{k(f-B)}\right)>1$ for any solution, which in view of the equivalence of (2.26) with the equality in (2.25) implies that $h \neq(1-k)^{1-q}(f-B)^{q}$ and $\frac{k^{1-q} B^{q}}{h-(1-k)^{1-q}(f-B)^{q}}>1$ and so since $B>0$ that also $h>(1-k)^{1-q}(f-B)^{q}$ for any such solution. Therefore any solution of (2.26) in $(0, f)$ will automatically belong to the interior of $W_{k}^{\prime}$ and would be a root of $R_{k}^{\prime}$ there.

Now letting $x=\frac{B}{k f} \in\left(0, \frac{1}{k}\right)$ the equation (2.26) takes the form (2.14) with $\lambda=\frac{f^{q}}{h}>1$, and hence it has always a unique solution $x=x_{\lambda}(k)$ in $\left(1, \frac{1}{k}\right)$; hence (2.26) has a unique solution $B^{*}=x_{\lambda}(k) k f$ in $(k f, f)$ and it has a solution in $(0,1)$ if and only if $\frac{f^{q}}{h}=\lambda<(1-k)^{q-1}$, which is unique. Hence (2.26) has a unique solution $B_{0}$ in $(0, k f)$ if $(1-k)^{1-q} f^{q}<h$ and it has no solutions there otherwise. Moreover by the above remarks, $B^{*}$ satisfies (2.22).

If $(1-k)^{1-q} f^{q}<h$, then $\eta_{0}=0<\rho_{0}$ and so $\lim _{B \rightarrow \rho_{0}^{+}} R_{k}^{\prime}(B)=+\infty, R_{k}^{\prime}(k f)>0$ and $\lim _{B \rightarrow \rho_{1}^{-}} R_{k}^{\prime}(B)=-\infty$, which imply that the maximum value of $R_{k}$ is attained exactly at $B^{*}>k f\left(B_{0}<k f\right.$ in this case must be an inflection point).

If $(1-k)^{1-q} f^{q} \geq h>(1-k) f^{q}$, then $B^{*}$ is the unique root of $R_{k}^{\prime}$ in the interior of $W_{k}^{\prime}$ and so $R_{k}^{\prime}(k f)>0$ and $\lim _{B \rightarrow \rho_{1}^{-}} R_{k}^{\prime}(B)=-\infty$ imply that the maximum value of $R_{k}$ is attained exactly at $B^{*}$.

If $(1-k) f^{q} \geq h$, then $f \geq \rho_{1}>\eta_{0} \geq k f>\rho_{0}$ and $\lim _{B \rightarrow \eta_{0}^{+}} Z(B)=+\infty$, so since $q<1$ we get from (2.24) that $\lim _{B \rightarrow \eta_{0}^{+}} R_{k}^{\prime}(B)=\frac{q}{1-q}\left(\frac{\eta_{0}}{k}\right)^{q-1}>0$. Again the maximum value of $R_{k}$ is attained exactly at $B^{*}$. 
To complete the proof of the lemma we observe that since $\omega_{q}\left(Z\left(B^{*}\right)\right)^{1 / q}=$ $\frac{B^{*}(1-k)}{k\left(f-B^{*}\right)}$

$$
\begin{aligned}
R_{k}\left(B^{*}\right) & =h \omega_{q}\left(Z\left(B^{*}\right)\right)-(1-k)^{1-q}\left(f-B^{*}\right)^{q}\left(\frac{B^{*}(1-k)}{k\left(f-B^{*}\right)}\right)^{q} \\
& =h \omega_{q}\left(Z\left(B^{*}\right)\right)-(1-k)\left(\frac{B^{*}}{k}\right)^{q}
\end{aligned}
$$

and then use $B^{*}=x_{\lambda}(k) k f$ and $Z\left(B^{*}\right)=H_{q}\left(\frac{B^{*}(1-k)}{k\left(f-B^{*}\right)}\right)=\lambda H_{q}\left(x_{\lambda}(k)\right)$.

\section{TREES AND MAXIMAL OPERATORS}

As in 4 we let $(X, \mu)$ be a nonatomic probability space (i.e. $\mu(X)=1$ ). Two measurable subsets $A, B$ of $X$ will be called almost disjoint if $\mu(A \cap B)=0$. Then we give the following.

Definition 1. A set $\mathcal{T}$ of measurable subsets of $X$ will be called a tree if the following conditions are satisfied:

(i) $X \in \mathcal{T}$ and for every $I \in \mathcal{T}$ we have $\mu(I)>0$.

(ii) For every $I \in \mathcal{T}$ there corresponds a finite or countable subset $\mathcal{C}(I) \subseteq \mathcal{T}$ containing at least two elements such that:

(a) the elements of $\mathcal{C}(I)$ are pairwise almost disjoint subsets of $I$,

(b) $I=\bigcup \mathcal{C}(I)$.

(iii) $\mathcal{T}=\bigcup_{m \geq 0} \mathcal{T}_{(m)}$, where $\mathcal{T}_{(0)}=\{X\}$ and $\mathcal{T}_{(m+1)}=\bigcup_{I \in \mathcal{T}_{(m)}} \mathcal{C}(I)$.

(iv) We have $\lim _{m \rightarrow \infty} \sup _{I \in \mathcal{T}_{(m)}} \mu(I)=0$.

For any tree $\mathcal{T}$ we define its exceptional set $E=E(\mathcal{T})$ as follows:

$$
E(\mathcal{T})=\bigcup_{I \in \mathcal{T}} \bigcup_{\substack{J_{1}, J_{2} \in \mathcal{C}(I) \\ J_{1} \neq J_{2}}}\left(J_{1} \cap J_{2}\right)
$$

It is clear that $E(\mathcal{T})$ has measure 0 .

An easy induction shows that each family $\mathcal{T}_{(m)}$ consists of pairwise almost disjoint sets whose union is $X$. Moreover if $x \in X \backslash E(\mathcal{T})$, then for each $m$ there exists exactly one $I_{m}(x)$ in $\mathcal{T}_{(m)}$ containing $x$. For every $m>0$ there is a $J \in \mathcal{T}_{(m-1)}$ such that $I_{m}(x) \in \mathcal{C}(J)$. Since then $x \in J$ we must have $J=I_{m-1}(x)$. Hence the set $\mathcal{A}(x)=\{I \in \mathcal{T}: x \in I\}$ forms a chain $I_{0}(x)=X \supseteq I_{1}(x) \supseteq \ldots$ with $I_{m}(x) \in \mathcal{C}\left(I_{m-1}(x)\right)$ for every $m>0$. From this remark it easily follows that if $I, J \in \mathcal{T}$ and $I \cap J \cap(X \backslash E(\mathcal{T}))$ is nonempty, then $I \subseteq J$ or $J \subseteq I$. In particular for any $I, J \in \mathcal{T}$ we have either $\mu(I \cap J)=0$ or one of them is contained in the other. The following gives another property of $\mathcal{T}$ that will be useful later. For a proof see 4].

Lemma 5. For every $I \in \mathcal{T}$ and every $\alpha$ such that $0<\alpha<1$ there exists a subfamily $\mathcal{F}(I) \subseteq \mathcal{T}$ consisting of pairwise almost disjoint subsets of I such that

$$
\mu\left(\bigcup_{J \in \mathcal{F}(I)} J\right)=\sum_{J \in \mathcal{F}(I)} \mu(J)=(1-\alpha) \mu(I) .
$$


Now given any tree $\mathcal{T}$ we define the maximal operator associated to it as follows:

$$
M_{\mathcal{T}} \phi(x)=\sup \left\{\frac{1}{\mu(I)} \int_{I}|\phi| d \mu: x \in I \in \mathcal{T}\right\}
$$

for every $\phi \in L^{1}(X, \mu)$.

The linearization of this operator we will use has been introduced in [4. Here we will recall its definition and the properties that we will use (for more details, see [4]).

Let $\phi \in L^{1}(X, \mu)$ be a nonnegative function and for any $I \in \mathcal{T}$ let

$$
\operatorname{Av}_{I}(\phi)=\frac{1}{\mu(I)} \int_{I} \phi d \mu
$$

We will say that $\phi$ is $\mathcal{T}$-good if the set $\Lambda_{\phi}=\left\{x \in X \backslash E(\mathcal{T}): M_{\mathcal{T}} \phi(x)>\operatorname{Av}_{I}(\phi)\right.$ for all $I \in \mathcal{T}$ such that $x \in I\}$ has $\mu$-measure zero. If $m \geq 0$ and $\lambda_{I} \geq 0$ for each $I \in \mathcal{T}_{(m)}$ are given, then the function $\phi=\sum_{I \in \mathcal{T}_{(m)}} \lambda_{I} \chi_{I}$ (where $\chi_{I}$ denotes the characteristic function of $I)$ is $\mathcal{T}$-good since $\operatorname{Av}_{J}(\phi)=\operatorname{Av}_{P}(\phi)$ whenever $J \in \mathcal{C}(P)$, $P \in \mathcal{T}_{(s)}$ for $s>m$ and so $\Lambda_{\phi}=\varnothing$. We call such a $\phi$ a $\mathcal{T}$-step function. However not all functions are $\mathcal{T}$-good but $\mathcal{T}$-step functions will be enough for our purposes.

Suppose now that $\phi$ is $\mathcal{T}$-good. Then for any $x \in X \backslash\left(E(\mathcal{T}) \cup \Lambda_{\phi}\right)$ (i.e., for $\mu$-almost every $x$ in $X$ ) we define $I_{\phi}(x)$ to be the largest element in the nonempty set $\left\{I \in \mathcal{T}: x \in I\right.$ and $\left.M_{\mathcal{T}} \phi(x)=\operatorname{Av}_{I}(\phi)\right\}$. let

Also given any $I \in \mathcal{T}$ let $A(\phi, I)=\left\{x \in X \backslash\left(E(\mathcal{T}) \cup \Lambda_{\phi}\right): I_{\phi}(x)=I\right\} \subseteq I$ and

$$
\mathcal{S}_{\phi}=\{I \in \mathcal{T}: \mu(A(\phi, I))>0\} \cup\{X\},
$$

which is a subtree of $\mathcal{T}$. We also define the correspondence $I \rightarrow I^{*}$ with respect to $\mathcal{S}_{\phi}$ as follows: $I^{*}$ is the smallest element of $\left\{J \in \mathcal{S}_{\phi}: I \varsubsetneqq J\right\}$. This is defined for every $I$ in $\mathcal{S}_{\phi}$ except $X$. It is clear that the $A(\phi, I)$ 's for $I \in \mathcal{S}_{\phi}$ are pairwise disjoint and their union has full measure. Their basic properties are the following, $A \approx B$ meaning $\mu(A \backslash B)=\mu(B \backslash A)=0$ (see [4]):

(i) If $I, J \in \mathcal{S}_{\phi}$, then either $A(\phi, J) \cap I=\varnothing$ or $J \subseteq I$.

(ii) If $I \in \mathcal{S}_{\phi}$, then there exists $J \in \mathcal{C}(I)$ such that $J \notin \mathcal{S}_{\phi}$.

(iii) For every $I \in \mathcal{S}_{\phi}$ we have $I \approx \bigcup_{\mathcal{S}_{\phi} \ni J \subseteq I} A(\phi, J)$.

(iv) For every $I \in \mathcal{S}_{\phi}$ we have $A(\phi, I) \approx I \backslash \bigcup_{J \in \mathcal{S}_{\phi}: J^{*}=I} J$ and so

$$
\mu(A(\phi, I))=\mu(I)-\sum_{J \in \mathcal{S}: J^{*}=I} \mu(J) .
$$

In particular (iv) implies

$$
\operatorname{Av}_{I}(\phi)=\frac{1}{\mu(I)} \sum_{J \in \mathcal{S}_{\phi}: J \subseteq I} \int_{A(\phi, J)} \phi d \mu,
$$

so setting

$$
a_{I}=\mu(A(\phi, I)) \text { and } x_{I}=a_{I}^{-1} \int_{A(\phi, I)} \phi d \mu
$$


for every $I \in \mathcal{S}_{\phi}$ (in the case where $\mu(A(\phi, X))=0$ we set $x_{X}=0$ ) we have

$$
M_{\mathcal{T}} \phi=\sum_{I \in \mathcal{S}_{\phi}}\left(\frac{1}{\mu(I)} \sum_{J \in \mathcal{S}_{\phi}, J \subseteq I} a_{J} x_{J}\right) \chi_{A(\phi, I)}
$$

and

$$
\int_{X} \phi d \mu=\sum_{I \in \mathcal{S}_{\phi}} \int_{A(\phi, I)} \phi d \mu=\sum_{I \in \mathcal{S}_{\phi}} a_{I} x_{I} .
$$

Using this decomposition of $M_{\mathcal{T}} \phi$ we can now prove the following:

Lemma 6. If the nonnegative function $\phi \in L^{1}(X, \mu)$ is $\mathcal{T}$-good, $\int_{X} \phi d \mu=f$ and $\int_{X} \phi^{q} d \mu=h$, then we have

$$
\int_{X}\left(M_{\mathcal{T}} \phi\right)^{q} d \mu \leq h \omega_{q}\left(\frac{f^{q}}{h}\right) .
$$

Proof. We let $\mathcal{S}=\mathcal{S}_{\phi}, a_{I}=\mu(A(\phi, I))$,

$$
\rho_{I}=\frac{a_{I}}{\mu(I)} \in(0,1)
$$

(except possibly for $I=X$ ) and

$$
y_{I}=\operatorname{Av}_{I}(\phi)=\frac{1}{\mu(I)} \sum_{J \in \mathcal{S}: J \subseteq I} a_{J} x_{J}
$$

for every $I \in \mathcal{S}$. It is easy to see that

$$
y_{I} \mu(I)=\sum_{J \in \mathcal{S}: J^{*}=I} y_{J} \mu(J)+a_{I} x_{I}
$$

and so using the concavity of the function $t \rightarrow t^{q}$ we have for any $I \in \mathcal{S}$,

$$
\begin{aligned}
\left(y_{I} \mu(I)\right)^{q} & =\left(\sum_{J \in \mathcal{S}: J^{*}=I} \tau_{I} \mu(J) \frac{y_{J}}{\tau_{I}}+\sigma_{I} a_{I} \frac{x_{I}}{\sigma_{I}}\right)^{q} \\
& \geq \sum_{J \in \mathcal{S}: J^{*}=I} \tau_{I} \mu(J)\left(\frac{y_{J}}{\tau_{I}}\right)^{q}+\sigma_{I} a_{I}\left(\frac{x_{I}}{\sigma_{I}}\right)^{q}
\end{aligned}
$$

whenever the $\tau_{I}, \sigma_{I}>0$ satisfy $\tau_{I}\left(\mu(I)-a_{I}\right)+\sigma_{I} a_{I}=\sum_{J \in \mathcal{S}: J^{*}=I} \tau_{I} \mu(J)+\sigma_{I} a_{I}=1$.

We now fix $\beta>0$ and let

$$
\sigma_{I}=\left((\beta+1) \mu(I)-\beta a_{I}\right)^{-1} \text { and } \tau_{I}=(\beta+1) \sigma_{I},
$$

which satisfy the above relation to get, dividing by $\sigma_{I}^{1-q}$,

$$
\left((\beta+1) \mu(I)-\beta a_{I}\right)^{1-q}\left(y_{I} \mu(I)\right)^{q} \geq \sum_{J \in \mathcal{S}: J^{*}=I}(\beta+1)^{1-q} \mu(J) y_{J}^{q}+a_{I} x_{I}^{q} .
$$

However $x_{I}^{q}=\left(\frac{1}{a_{I}} \int_{A(\phi, I)} \phi\right)^{q} \geq \frac{1}{a_{I}} \int_{A(\phi, I)} \phi^{q}$ and so summing over all $I \in \mathcal{S}$ (if $a_{X}=0$, then $\left.\mu(A(\phi, X))=0\right)$ and using the properties of the $A(\phi, I)$ 's we get

$$
\sum_{I \in \mathcal{S}}\left((\beta+1) \mu(I)-\beta a_{I}\right)^{1-q}\left(y_{I} \mu(I)\right)^{q} \geq \sum_{\substack{J \in \mathcal{S} \\ J \neq X}}(\beta+1)^{1-q} \mu(J) y_{J}^{q}+\int_{X} \phi^{q} d \mu
$$

and so since $y_{X}=f$ we get

$$
(\beta+1)^{1-q} f^{q}-h \geq \sum_{I \in \mathcal{S}}\left[(\beta+1)^{1-q} \mu(I)-\left((\beta+1) \mu(I)-\beta a_{I}\right)^{1-q}(\mu(I))^{q}\right] y_{I}^{q},
$$


which since

$$
\begin{aligned}
& \frac{1}{\mu(I)}\left[(\beta+1)^{1-q} \mu(I)-\left((\beta+1) \mu(I)-\beta a_{I}\right)^{1-q}(\mu(I))^{q}\right] \\
= & (\beta+1)^{1-q}-\left((\beta+1)-\beta \rho_{I}\right)^{1-q} \geq(1-q)(\beta+1)^{-q} \beta \rho_{I}
\end{aligned}
$$

gives

$$
(\beta+1)^{1-q} f^{q}-h \geq(1-q)(\beta+1)^{-q} \beta \sum_{I \in \mathcal{S}} a_{I} y_{I}^{q}
$$

and so for any $\beta>0$ we have

$$
\int_{X}\left(M_{\mathcal{T}} \phi\right)^{q} d \mu \leq \frac{1}{1-q} \frac{(\beta+1) f^{q}-(\beta+1)^{q} h}{\beta} .
$$

Considering now the right-hand side of (3.18) as a function of $\beta$ it is easy to see that it is minimized for $\beta$ equal to the unique root of the equation

$$
H_{q}(\beta+1)=(1-q)(\beta+1)^{q}+q(\beta+1)^{q-1}=\frac{f^{q}}{h} \geq 1
$$

and so taking $\beta=\omega_{q}\left(\frac{f^{q}}{h}\right)^{1 / q}-1 \geq 0$ in (3.18) and using (3.19) completes the proof of (3.11).

Next we show that (3.11) holds for general $\phi$ and that it is actually best possible. This is the content of the following.

Theorem 2. For any $0<q<1$ we have

$$
\sup \left\{\int_{X}\left(M_{\mathcal{T}} \phi\right)^{q} d \mu: \phi \geq 0, \phi \in L^{1}(X, \mu), \int_{X} \phi d \mu=f, \int_{X} \phi^{q} d \mu=h\right\}=h \omega_{q}\left(\frac{f^{q}}{h}\right) .
$$

Proof. For the general nonnegative $\phi \in L^{1}(X, \mu)$ one can consider the sequence $\left(\phi_{m}\right)$, where $\phi_{m}=\sum_{I \in \mathcal{T}_{(m)}} \operatorname{Av}_{I}(\phi) \chi_{I}$, and set

$$
\Phi_{m}=\sum_{I \in \mathcal{T}_{(m)}} \max \left\{\operatorname{Av}_{J}(\phi): I \subseteq J \in \mathcal{T}\right\} \chi_{I}=M_{\mathcal{T}} \phi_{m}
$$

since $\operatorname{Av}_{J}(\phi)=\operatorname{Av}_{J}\left(\phi_{m}\right)$ whenever $I \subseteq J \in \mathcal{T}$ when $I \in \mathcal{T}_{(m)}$.

Then it is easy to see that

$$
\int_{X} \phi_{m} d \mu=\int_{X} \phi d \mu=f, h_{m}=\int_{X} \phi_{m}^{q} d \mu \geq \int_{X} \phi^{q} d \mu=h
$$

for all $m$ and that $\Phi_{m}$ converges monotonically almost everywhere to $M_{\mathcal{T}} \phi$. Since as we have seen, each $\phi_{m}$ is $\mathcal{T}$-good, (3.11) combined with Lemma 1 (iii) gives

$$
\int_{X} \Phi_{m}^{q} d \mu \leq h_{m} \omega_{q}\left(\frac{f^{q}}{h_{m}}\right) \leq h \omega_{q}\left(\frac{f^{q}}{h}\right)
$$

and so letting $m \rightarrow \infty$ we get (3.11) for the general $\phi$.

Now to complete the proof of the theorem we use a construction similar to [4, and for $\alpha$ with $0<\alpha<1$, using Lemma 5 , for every $I \in \mathcal{T}$ we choose a family $\mathcal{F}(I) \subseteq \mathcal{T}$ of pairwise almost disjoint subsets of $I$ such that

$$
\sum_{J \in \mathcal{F}(I)} \mu(J)=(1-\alpha) \mu(I) .
$$


Then we define $\mathcal{S}=\mathcal{S}_{\alpha}$ to be the smallest subset of $\mathcal{T}$ such that $X \in \mathcal{S}$ and for every $I \in \mathcal{S}, \mathcal{F}(I) \subseteq \mathcal{S}$. It is clear that defining the correspondence $I \rightarrow I^{*}$ with respect to this $\mathcal{S}$ we have $J^{*}=I \in \mathcal{S}$ if and only if $J \in \mathcal{F}(I)$ and so writing

$$
A_{I}=I \backslash \bigcup_{J \in \mathcal{S}: J^{*}=I} J
$$

we have $a_{I}=\mu\left(A_{I}\right)=\mu(I)-\sum_{J \in \mathcal{S}: J^{*}=I} \mu(J)=\alpha \mu(I)$ for every $I \in \mathcal{S}$.

Also it is easy to see that

$$
\mathcal{S}=\bigcup_{m \geq 0} \mathcal{S}_{(m)}, \text { where } \mathcal{S}_{(0)}=\{X\} \text { and } \mathcal{S}_{(m+1)}=\bigcup_{I \in \mathcal{S}_{(m)}} \mathcal{F}(I) .
$$

We define the rank $r(I)$ of any $I \in \mathcal{S}$ to be the unique integer $m$ such that $I \in \mathcal{S}_{(m)}$ and for $\lambda, \gamma>0$ to be chosen later we define the $x_{I}$ 's by setting

$$
x_{I}=\lambda \gamma^{r(I)}
$$

for every $I \in \mathcal{S}$. For every $I \in \mathcal{S}$ and every $m \geq 0$ we have

$$
b_{m}(I)=\sum_{\substack{\mathcal{S} \ni \ni \subseteq I \\ r(J)=r(\bar{I})+m}} \mu(J)=(1-\alpha)^{m} \mu(I) ;
$$

hence

$$
\begin{aligned}
\sum_{I \in \mathcal{S}} a_{I} x_{I}^{r} & =\lambda^{r} \sum_{m \geq 0} \sum_{I \in \mathcal{S}_{(m)}} \gamma^{m r} \alpha \mu(J)=\lambda^{r} \alpha \sum_{m \geq 0} \gamma^{m r} b_{m}(X) \\
& =\lambda^{r} \alpha \sum_{m \geq 0}\left[\gamma^{r}(1-\alpha)\right]^{m}=\frac{\lambda^{r} \alpha}{1-\gamma^{r}(1-\alpha)}
\end{aligned}
$$

for any $r>0$ such that $\gamma^{r}(1-\alpha)<1$. On the other hand, if $\gamma(1-\alpha)<1$,

$$
\begin{aligned}
y_{I} & =\frac{1}{\mu(I)} \sum_{J \in \mathcal{S}: J \subseteq I} a_{J} x_{J}=\frac{\lambda}{\mu(I)} \sum_{\mathcal{S} \ni J \subseteq I} \alpha \mu(J) \gamma^{r(J)} \\
& =\frac{\lambda \alpha}{\mu(I)} \sum_{m \geq 0} \gamma^{m+r(I)} \sum_{\substack{\mathcal{S} \ni J \subseteq I \\
r(J)=r(I)+m}} \mu(J) \\
& =\lambda \alpha \gamma^{r(I)} \sum_{m \geq 0} \gamma^{m}(1-\alpha)^{m}=\frac{\alpha}{1-\gamma(1-\alpha)} x_{I},
\end{aligned}
$$

where the $y_{I}$ 's are defined by the first equality above and so

$$
\sum_{I \in \mathcal{S}} a_{I} y_{I}^{q}=\left(\frac{\alpha}{1-\gamma(1-\alpha)}\right)^{q} \sum_{I \in \mathcal{S}} \alpha_{I} x_{I}^{q}
$$

whereas

$$
y_{X}=\frac{\lambda \alpha}{1-\gamma(1-\alpha)} .
$$

We choose $\lambda=f \alpha^{-1}(1-\gamma(1-\alpha))$ to make $y_{X}=f$ and $\gamma=\frac{z-\alpha}{z(1-\alpha)}$, where $z=z_{q}\left(\alpha, \frac{f^{q}}{h}\right)>1$ is the unique root of $\frac{z^{q}-(1-\alpha)^{1-q}(z-\alpha)^{q}}{\alpha}=\frac{f^{q}}{h}$ furnished by Lemma 2. Obviously $\gamma>1$ and

$$
\gamma^{q}(1-\alpha)<\gamma(1-\alpha)=1-\frac{\alpha}{z}<1
$$


and so we have for these choices of $\lambda, \gamma$,

$$
\sum_{I \in \mathcal{S}} x_{I}^{q}=h, y_{X}=f \text { and } \sum_{I \in \mathcal{S}} a_{I} y_{I}^{q}=z_{q}\left(\alpha, \frac{f^{q}}{h}\right)^{q} F .
$$

Next we consider the function

$$
\phi_{\alpha}=\sum_{I \in \mathcal{S}} x_{I} \chi_{A_{I}}
$$

so that $\int_{X} \phi_{\alpha} d \mu=f, \int_{X} \phi_{\alpha}^{q} d \mu=h$, and since $M_{\mathcal{T}} \phi_{\alpha} \geq \sum_{I \in \mathcal{S}} \operatorname{Av}_{I}\left(\phi_{\alpha}\right) \chi_{A_{I}}$ we have

$$
\int_{X}\left(M_{\mathcal{T}} \phi_{\alpha}\right)^{q} d \mu \geq \sum_{I \in \mathcal{S}} a_{I} y_{I}^{q}=z\left(\alpha, \frac{f^{q}}{h}\right)^{q} F .
$$

Now we let $\alpha \rightarrow 0^{+}$and use Lemma 2 to complete the proof.

Now using Lemma 1 (ii) in (3.20) we get

$$
\int_{X}\left(M_{\mathcal{T}} \phi\right)^{q} d \mu<\frac{1}{1-q}\left(\int_{X} \phi d \mu\right)^{q}
$$

which is what Kolmogorov's inequality would imply. However, since by Lemma 1(ii), $\lim _{h \rightarrow 0^{+}} h \omega_{q}\left(\frac{f^{q}}{h}\right)=\frac{f^{q}}{1-q}$ the above theorem implies that (3.33) is actually sharp. When the function $\phi$ satisfies certain additional properties the above proof also gives the following improvement (which shows Lemma 2 for $z_{q}$ ).

Theorem 3. Let $0<q<1$ and suppose $\alpha$ is such that $0<\alpha<1$. If $\phi \geq 0$ is $\mathcal{T}$-good with $\int_{X} \phi d \mu=f, \int_{X} \phi^{q} d \mu=h$ and such that $a_{I}=\mu(A(\phi, I)) \geq \alpha \mu(I)$ for every $I \in \mathcal{S}=\mathcal{S}_{\phi}$, then

$$
\int_{X}\left(M_{\mathcal{T}} \phi\right)^{q} d \mu \leq z_{q}\left(\alpha, \frac{f^{q}}{h}\right) h<\frac{\alpha}{1-(1-\alpha)^{1-q}} f^{q}<\frac{1}{1-q} f^{q}
$$

and the first of these inequalities is best possible.

Proof. To prove (3.34) we use with $\rho_{I}=\frac{a_{I}}{\mu(I)} \geq \alpha$ the inequality

$$
\frac{(\beta+1)^{1-q}-\left((\beta+1)-\beta \rho_{I}\right)^{1-q}}{\rho_{I}} \geq \frac{(\beta+1)^{1-q}-((\beta+1)-\beta \alpha)^{1-q}}{\alpha}
$$

in (3.17) to get for any $\beta>0$,

$$
\int_{X}\left(M_{\mathcal{T}} \phi\right)^{q} d \mu \leq \alpha \frac{(\beta+1)^{1-q} f^{q}-h}{(\beta+1)^{1-q}-(\beta+1-\beta \alpha)^{1-q}}
$$

instead of (3.18). Now setting $z=\beta+1-\beta \alpha>1$ it is easy to see that the righthand side is minimized when $z$ satisfies (2.5) with $\lambda=\frac{f^{q}}{h}$ and so using Lemma 2 and the equation (2.5) in (3.35) completes the proof of (3.34).

To show that (3.34) is best possible we just use the $\phi_{\alpha}$ in the proof of Theorem 2 for this specific $\alpha$.

The above theorem has the following application in the case where $X=[0,1]^{n}, \mu$ is the Lebesgue measure and $\mathcal{T}=\mathcal{D}$ is the tree of all dyadic subcubes of $[0,1]^{n}$ and therefore $\mathcal{D}_{(m)}$ is the set of all dyadic subcubes of $[0,1]^{n}$ whose sides have length equal to $2^{-m}$. 
Proposition 2. Let $0<q<1$. Then:

(i) If $m>0$ and $\phi=\sum_{I \in \mathcal{D}_{(m)}} \lambda_{I} \chi_{I}$, then (with $\int_{X} \phi d \mu=f, \int_{X} \phi^{q} d \mu=h$ )

$$
\int_{X}\left(M_{\mathcal{D}} \phi\right)^{q} d \mu \leq z_{q}\left(2^{-m n}, \frac{f^{q}}{h}\right) h<\frac{1}{2^{m n}-2^{m n q}\left(2^{m n}-1\right)^{1-q}}\|\phi\|_{1}^{q} .
$$

(ii) If $\phi \in L^{1}$ and is nonnegative, decreasing in each variable and convex on $Q=(0,1)^{n}$, then

$$
\int_{Q}\left(M_{d} \phi\right)^{q} \leq z_{q}\left(2^{-n}, \frac{f^{q}}{h}\right) h<\frac{1}{2^{n}-2^{n q}\left(2^{n}-1\right)^{1-q}}\|\phi\|_{1}^{q} .
$$

Proof. Both follow from (3.34). For (3.36) it suffices to observe that $\phi$ is $\mathcal{D}$-good and for any $J \in \mathcal{S}_{\phi}$ we choose $I \in \mathcal{D}_{(m)}$ such that $|A(\phi, J) \cap I|>0$ and note that we must have $I \subseteq A(\phi, J)$ and so $\frac{|A(\phi, J)|}{|J|} \geq 2^{-m n}$.

For (3.37) we use (3.34) for $\alpha=\frac{1}{2^{n}}$ noting that for any $J \in \mathcal{D}$ if $J_{+}$is the square formed by the right halves of the sides of $J$, the assumptions on $\phi$ imply

$$
\operatorname{Av}_{J}(\phi) \geq \sup _{x \in J_{+}} \phi(x)
$$

and therefore $\phi$ is $\mathcal{D}$-good and for any $J \in \mathcal{S}_{\phi}$ we have $J_{+} \subseteq A(\phi, J)$.

The first part of the above proposition provides an estimate of the limitation for making the sharp inequality (1.3) an almost equality using dyadic step functions.

\section{Bellman Functions FOR the maXimal operator RElated to KOLMOGOROV'S INEQUALITY}

Here we use the results of the previous sections to study the Bellman functions for $M_{\mathcal{T}}$ defined by (1.6), thus proving our main Theorem 1 .

Let us fix $0<q<1$ and a nonnegative $\phi \in L^{1}(X, \mu)$ such that $\int_{X} \phi^{q} d \mu=h$ and $\int_{X} \phi d \mu=f$ (where $h \leq f^{q}$ ). For any $I \in \mathcal{T}$ we apply Theorem 3 for $\phi$ restricted to $I$ and for the tree $\mathcal{T}(I)=\{J \in \mathcal{T}: J \subseteq I\}$ on the probability space $\left(I, \frac{1}{\mu(I)} \mu\right)$ to get

$$
\operatorname{Av}_{I}\left[\left(M_{\mathcal{T}(I)}\left(\phi \chi_{I}\right)\right)^{q}\right] \leq \operatorname{Av}_{I}\left(\phi^{q}\right) \omega_{q}\left(\frac{\left(\operatorname{Av}_{I}(\phi)\right)^{q}}{\operatorname{Av}_{I}\left(\phi^{q}\right)}\right) .
$$

Next fix $L \geq f$ and let $I_{1}, I_{2}, \ldots$ be all the maximal elements (if any) of $\{J \in \mathcal{T}$ : $\left.\operatorname{Av}_{J}(\phi) \geq L\right\}$. It is clear that the $I_{j}$ 's are pairwise almost disjoint and that writing $K=\bigcup_{j} I_{j}$

$$
\max \left(M_{\mathcal{T}} \phi, L\right)=L \chi_{X \backslash K}+\sum_{j}\left(M_{\mathcal{T}\left(I_{j}\right)}\left(\phi \chi_{I_{j}}\right)\right) \chi_{I_{j}} .
$$

Therefore writing $k=\sum_{j} \mu\left(I_{j}\right)$ and using (4.1) for $I_{1}, I_{2}, \ldots$ we get

$$
\int_{X}\left(\max \left(M_{\mathcal{T}} \phi, L\right)\right)^{q} d \mu \leq L^{q}(1-k)+\sum_{j} \alpha_{j} \omega_{q}\left(\frac{\beta_{j}}{\alpha_{j}}\right)^{q},
$$


where

$$
\alpha_{j}=\int_{I_{j}} \phi^{q} d \mu \leq \beta_{j}=\mu\left(I_{j}\right)^{1-q}\left(\int_{I_{j}} \phi d \mu\right)^{q}
$$

Now we write

$$
A=\sum_{j} \alpha_{j}=\int_{K} \phi^{q} d \mu \leq h \text { and } B=\sum_{j}\left(\mu\left(I_{j}\right)^{q-1} \beta_{j}\right)^{1 / q}=\int_{K} \phi d \mu \leq f,
$$

note that

$$
k^{1-q} B^{q}=\left(\sum_{j} \mu\left(I_{j}\right)\right)^{1-q}\left(\sum_{j}\left(\mu\left(I_{j}\right)^{q-1} \beta_{j}\right)^{1 / q}\right)^{q} \geq \sum_{j} \beta_{j} \geq A,
$$

and use the concavity of $\omega_{q}$ provided by Lemma 1 (i) to conclude that

$$
\begin{aligned}
\int_{X}\left(\max \left(M_{\mathcal{T}} \phi, L\right)\right)^{q} d \mu & \leq L^{q}(1-k)+A \omega_{q}\left(\frac{\sum_{j} \beta_{j}}{A}\right) \\
& \leq L^{q}(1-k)+A \omega_{q}\left(\frac{k^{1-q} B^{q}}{A}\right) .
\end{aligned}
$$

The parameters $A, B$ and $k$ satisfy the following inequalities:

$$
A \leq k^{1-q} B^{q}, A \leq h, B \leq f, 0 \leq k \leq 1 \text { and } h-A \leq(1-k)^{1-q}(f-B)^{q},
$$

the last one being just $\int_{X \backslash K} \phi^{q} d \mu \leq \mu(X \backslash K)^{1-q}\left(\int_{X \backslash K} \phi d \mu\right)^{q}$ (we also have $B \geq k L$ by the choice of the $I_{j}$ 's).

Conversely assuming that $0<k<1, B<f$ and $A, B$ satisfy the inequalities (4.8), we fix $\delta$ in $(0,1)$ and we use Lemma 5 to pick a family $\left\{I_{1}, I_{2}, \ldots\right\}$ of pairwise almost disjoint elements of $\mathcal{T}$ such that $\sum_{j} \mu\left(I_{j}\right)=k$. Next since $A \leq k^{1-q} B^{q}$, using Theorem 3 for each $j$ we choose a nonnegative $\phi_{j} \in L^{1}\left(I_{j}, \frac{1}{\mu\left(I_{j}\right)} \mu\right)$ such that

$$
\int_{I_{j}} \phi_{j}^{q} d \mu=\frac{A}{k} \mu\left(I_{j}\right), \int_{I_{j}} \phi_{j} d \mu=\frac{B}{k} \mu\left(I_{j}\right)
$$

and

$$
\int_{I_{j}}\left(M_{\mathcal{T}\left(I_{j}\right)}\left(\phi_{j}\right)\right)^{q} d \mu \geq \delta \frac{A}{k} \omega_{q}\left(\frac{k^{1-q} B^{q}}{A}\right) \mu\left(I_{j}\right) .
$$

Next we choose a $\psi \in L^{1}(X \backslash K, \mu)$ such that

$$
\int_{X \backslash K} \psi^{q} d \mu=h-A>0 \text { and } \int_{X \backslash K} \psi d \mu=f-B>0,
$$

where $K=\bigcup_{j} I_{j}$, which is possible by (4.8), and define

$$
\phi=\psi \chi_{X \backslash K}+\sum_{j} \phi_{j} \chi_{I_{j}} .
$$

Then $\int_{X} \phi d \mu=f \leq L, \int_{X} \phi^{q} d \mu=h$ and

$$
\int_{X}\left(\max \left(M_{\mathcal{T}} \phi, L\right)\right)^{q} d \mu \geq L^{q}(1-k)+\delta A \omega_{q}\left(\frac{k^{1-q} B^{q}}{A}\right)^{q} .
$$


Now letting $\delta \rightarrow 1^{-}$we have proved the following:

$$
\begin{gathered}
\mathcal{B}_{q}^{\mathcal{T}}(f, h, L, 1)=\sup \left\{L^{q}(1-k)+A \omega_{q}\left(\frac{k^{1-q} B^{q}}{A}\right): k^{1-q} B^{q} \geq A, A \leq h,\right. \\
\left.B<f, 0<k<1 \text { and }(1-k)^{1-q}(f-B)^{q} \geq h-A\right\} .
\end{gathered}
$$

The inequalities (4.8) for $k, A$ and $B$ imply that $(1-k)^{1-q}(f-B)^{q}+k^{1-q} B^{q} \geq h$ and $A \geq h-(1-k)^{1-q}(f-B)^{q}=C$ and so using now Lemma 1 (iii) we conclude that $A \omega_{q}\left(\frac{k^{1-q} B^{q}}{A}\right) \leq C \omega_{q}\left(\frac{k^{1-q} B^{q}}{C}\right)$ if $h>(1-k)^{1-q}(f-B)^{q}$ and

$$
A \omega_{q}\left(\frac{k^{1-q} B^{q}}{A}\right)<\lim _{z \rightarrow 0^{+}} z \omega_{q}\left(\frac{k^{1-q} B^{q}}{z}\right)=\frac{k^{1-q} B^{q}}{1-q}
$$

if $h \leq(1-k)^{1-q}(f-B)^{q}$. So using the notation of (2.20), (2.21) and Lemma 4 we have

$$
\begin{aligned}
\mathcal{B}_{q}^{\mathcal{T}}(f, h, L, 1) & =\sup \left\{R_{k, h}(B)+L^{q}(1-k): 0<k<1,0<B<f \text { and } l_{k}(B) \geq h\right\} \\
& =\sup _{0<k<1}\left[h \omega_{q}\left(\frac{f^{q}}{h} H_{q}\left(x\left(\frac{f^{q}}{h}, k\right)\right)\right)+\left(L^{q}-f^{q} x\left(\frac{f^{q}}{h}, k\right)^{q}\right)(1-k)\right],
\end{aligned}
$$

and so by Lemma 3 (iii) with $\xi=1$ and $\mu=\frac{L}{f}>1$ we have proved the following

Proposition 3. For any tree $\mathcal{T}$ on $(X, \mu)$ and any $0<q<1$ we have

$$
\mathcal{B}_{q}^{\mathcal{T}}(f, h, L, 1)=h \omega_{q}\left(\frac{f^{q}}{h} H_{q}\left(\frac{L}{f}\right)\right) .
$$

Next to compute $\mathcal{B}_{q}^{\mathcal{T}}(f, h, f, k)$ for $0<k<1$ in a way similar to [4, suppose that $\phi, E$ satisfy the requirements in the corresponding definition, choose $u>0$ such that

$$
\mu\left(\left\{M_{\mathcal{T}} \phi>u\right\}\right) \leq k \leq \mu\left(\left\{M_{\mathcal{T}} \phi \geq u\right\}\right)
$$

and choose a measurable $D$ such that $V_{1}=\left\{M_{\mathcal{T}} \phi>u\right\} \subseteq D \subseteq\left\{M_{\mathcal{T}} \phi \geq u\right\}=V_{2}$ and $\mu(D)=k$ (note that $\mu$ is assumed nonatomic). Since $M_{\mathcal{T}} \phi \leq u$ on $E \backslash V_{1}$ it is easy to see that

$$
\int_{E}\left(M_{\mathcal{T}} \phi\right)^{q} d \mu \leq \int_{D}\left(M_{\mathcal{T}} \phi\right)^{q} d \mu
$$

and defining $s \in[0,1]$ by $\mu(D)=s \mu\left(V_{1}\right)+(1-s) \mu\left(V_{2}\right)$ we also have (since $M_{\mathcal{T}} \phi=u$ on $\left.V_{2} \backslash V_{1}\right)$

$$
\int_{D}\left(M_{\mathcal{T}} \phi\right)^{q} d \mu=s \int_{V_{1}}\left(M_{\mathcal{T}} \phi\right)^{q} d \mu+(1-s) \int_{V_{2}}\left(M_{\mathcal{T}} \phi\right)^{q} d \mu
$$

Now since each of the $V_{1}, V_{2}$ is a union of families $\left\{I_{j}^{(1)}\right\},\left\{I_{r}^{(2)}\right\}$ consisting of pairwise almost disjoint elements maximal under $\operatorname{Av}_{I}(\phi)>u$ (resp. $\geq u$ ), we clearly have $M_{\mathcal{T}} \phi=M_{\mathcal{T}(I)} \phi$ for each of those I's. Arguing as in the case of $\mathcal{B}_{q}^{\mathcal{T}}(f, h, L, 1)$ and using (4.17) and (4.18), we have

$$
\int_{E}\left(M_{\mathcal{T}} \phi\right)^{q} d \mu \leq \sum_{j} s \alpha_{j}^{(1)} \omega_{q}\left(\frac{\beta_{j}^{(1)}}{\alpha_{j}^{(1)}}\right)+\sum_{r}(1-s) \alpha_{r}^{(2)} \omega_{q}\left(\frac{\beta_{r}^{(2)}}{\alpha_{r}^{(2)}}\right),
$$


where $\alpha_{j}^{(1)}=\int_{I_{j}^{(1)}} \phi^{q} d \mu \leq \beta_{j}^{(1)}=\mu\left(I_{j}^{(1)}\right)^{1-q}\left(\int_{I_{j}^{(1)}} \phi d \mu\right)^{q}, \alpha_{r}^{(2)}=\int_{I_{r}^{(2)}} \phi^{q} d \mu \leq \beta_{r}^{(2)}=$ $\mu\left(I_{r}^{(2)}\right)^{1-q}\left(\int_{I_{r}^{(2)}} \phi d \mu\right)^{q}$. Hence using Lemma 1 (i) we have

$$
\int_{K}\left(M_{\mathcal{T}} \phi\right)^{q} d \mu \leq A \omega_{q}\left(\frac{\sum_{j} s \beta_{j}^{(1)}+\sum_{r}(1-s) \beta_{r}^{(2)}}{A}\right),
$$

where

$$
A=\sum_{j} s \alpha_{j}^{(1)}+\sum_{r}(1-s) \alpha_{r}^{(2)}=s \int_{V_{1}} \phi^{q} d \mu+(1-s) \int_{V_{2}} \phi^{q} d \mu \leq h
$$

Setting

$$
B=s \int_{V_{1}} \phi d \mu+(1-s) \int_{V_{2}} \phi d \mu \leq f
$$

and noting that $k=\mu(D)=\sum_{j} s \mu\left(I_{j}^{(1)}\right)+\sum_{r}(1-s) \mu\left(I_{r}^{(2)}\right)$ it is easy to see using (3.15) that

$$
k^{1-q} B^{q} \geq \sum_{j} s \beta_{j}^{(1)}+\sum_{r}(1-s) \beta_{r}^{(2)} \geq A
$$

and so since $\omega_{q}$ is increasing we have

$$
\int_{E}\left(M_{\mathcal{T}} \phi\right)^{q} d \mu \leq A \omega_{q}\left(\frac{k^{1-q} B^{q}}{A}\right) .
$$

Moreover we note that $A, B$ satisfy all the inequalities in (4.8) ( $k$ now being fixed), the last now being just

$$
\int_{X} \phi^{q} \eta d \mu \leq\left(\int_{X} \eta d \mu\right)^{1-q}\left(\int_{X} \phi \eta d \mu\right)^{q},
$$

where $\eta=s \chi_{X \backslash V_{1}}+(1-s) \chi_{X \backslash V_{2}}$.

In the same way as in the case for $\mathcal{B}_{q}^{\mathcal{T}}(f, h, L, 1)$ and using, for any $A, B$ satisfying (4.8) (with $k$ fixed), the functions $\phi$ defined by (4.12) we get the following:

$$
\mathcal{B}_{q}^{\mathcal{T}}(f, h, f, k)=\sup \left\{R_{k}(B): 0 \leq B \leq f \text { and } l_{k}(B) \geq h\right\},
$$

where $l_{k}$ and $R_{k}$ are defined by (2.20) and (2.21).

Hence Lemma 4 implies

Proposition 4. We have

$$
\mathcal{B}_{q}^{\mathcal{T}}(f, h, f, k)=h \omega_{q}\left(\frac{f^{q}}{h} H_{q}\left(x\left(\frac{f^{q}}{h}, k\right)\right)\right)-f^{q} x\left(\frac{f^{q}}{h}, k\right)^{q}(1-k) .
$$

Now to prove our main Theorem 1 we let $w>0$ be such that

$$
\mu\left(\left\{\max \left(M_{\mathcal{T}} \phi, L\right)>w\right\}\right) \leq k \leq \mu\left(\left\{\max \left(M_{\mathcal{T}} \phi, L\right) \geq w\right\}\right),
$$

choose a measurable set $K$ such that $U_{1}=\left\{\max \left(M_{\mathcal{T}} \phi, L\right)>w\right\} \subseteq K \subseteq$ $\left\{\max \left(M_{\mathcal{T}} \phi, L\right) \geq u\right\}=U_{2}$ and $\mu(K)=k$ and as with (4.17) we get

$$
\int_{E}\left(\max \left(M_{\mathcal{T}} \phi, L\right)\right)^{q} d \mu \leq \int_{K}\left(\max \left(M_{\mathcal{T}} \phi, L\right)\right)^{q} d \mu .
$$


We then consider two cases:

(i) If $w>L$, then $M_{\mathcal{T}} \phi>L$ on $K$ and so (4.29) and $\mu(K)=k$ give

$$
\int_{E}\left(\max \left(M_{\mathcal{T}} \phi, L\right)\right)^{q} d \mu \leq \mathcal{B}_{q}^{\mathcal{T}}(f, h, f, k) .
$$

(ii) If $w=L$, then let $\mu\left(U_{1}\right)=k_{1} \leq k$ and note that $M_{\mathcal{T}} \phi \leq L$ on $K \backslash U_{1}$ to get

$$
\begin{aligned}
\int_{E}\left(\max \left(M_{\mathcal{T}} \phi, L\right)\right)^{q} d \mu & \leq \int_{U_{1}}\left(M_{\mathcal{T}} \phi\right)^{q} d \mu+\int_{K \backslash U_{1}} L^{q} d \mu \\
& \leq \mathcal{B}_{q}^{\mathcal{T}}\left(f, h, f, k_{1}\right)+L^{q}\left(k-k_{1}\right) .
\end{aligned}
$$

Conversely given $0<k_{1} \leq k$ for any $0<\delta<1$ choose a $\tilde{\phi}$ satisfying the requirements and a measurable set $E_{1} \subseteq X$ with $\mu\left(E_{1}\right)=k_{1}$ that satisfy

$$
\int_{E_{1}}\left(M_{\mathcal{T}} \tilde{\phi}\right)^{q} d \mu \geq \delta \mathcal{B}_{q}^{\mathcal{T}}\left(f, h, f, k_{1}\right)
$$

and then choose a measurable $E_{2} \subseteq X \backslash E_{1}$ with $\mu\left(E_{2}\right)=k-k_{1}$. Then $E=E_{1} \cup E_{2}$ satisfies $\mu(E)=k$ and

$$
\int_{E}\left(\max \left(M_{\mathcal{T}} \tilde{\phi}, L\right)\right)^{q} d \mu \geq \int_{E_{1}}\left(M_{\mathcal{T}} \tilde{\phi}\right)^{q} d \mu+L^{q} \mu\left(E_{2}\right)=\delta \mathcal{B}_{q}^{\mathcal{T}}\left(f, h, f, k_{1}\right)+L^{q}\left(k-k_{1}\right) .
$$

Letting $\delta \rightarrow 1^{-}$we have proved that

$$
\mathcal{B}_{q}^{\mathcal{T}}(f, h, L, k)=\sup _{0<k_{1} \leq k}\left[\mathcal{B}_{q}^{\mathcal{T}}\left(f, h, f, k_{1}\right)+L^{q}\left(1-k_{1}\right)\right]-L^{q}(1-k) .
$$

But now (4.27) and Lemma 3 (iii) complete the proof of Theorem 1.

\section{Proof of Proposition 1}

We will show first that for any $I \in \mathcal{T}_{(1)}=\mathcal{C}(X)$ (see Definition 1)

$$
\limsup _{m \rightarrow \infty} \frac{1}{\mu(I)} \int_{I} \phi_{m} d \mu \leq f \text {. }
$$

Suppose not and choose an $I \in \mathcal{T}_{(1)}$ such that (5.1) is not satisfied and assume choosing a subsequence that

$$
\lim _{m \rightarrow \infty} \frac{1}{\mu(I)} \int_{I} \phi_{m} d \mu=f_{1}>f \text { and } 0 \leq \lim _{m \rightarrow \infty} \frac{1}{\mu(I)} \int_{I} \phi_{m}^{q} d \mu=h_{1} \leq f_{1}^{q} .
$$

Then letting $\tau=\mu(I) \in(0,1)$ we also have

$$
\begin{gathered}
0 \leq \lim _{m \rightarrow \infty} \frac{1}{\mu(X \backslash I)} \int_{X \backslash I} \phi_{m} d \mu=\frac{f-\tau f_{1}}{1-\tau}<f \text { and } \\
\lim _{m \rightarrow \infty} \frac{1}{\mu(X \backslash I)} \int_{X \backslash I} \phi_{m}^{q} d \mu=\frac{h-\tau h_{1}}{1-\tau} \geq 0 .
\end{gathered}
$$

Consider the trees $\mathcal{T}^{*}=\{J \in \mathcal{T}: J \subseteq I\}$ on the probability space $\left(I, \frac{1}{\tau} \mu\right)$ and $\mathcal{T}^{* *}=\{X \backslash I\} \cup\{J \in \mathcal{T}: J \subseteq X \backslash I\}$ on the probability space $\left(X \backslash I, \frac{1}{1-\tau} \mu\right)$ and note that in view of $f_{1}>f$ for all sufficiently large $m$ we have

$$
M_{\mathcal{T}} \phi_{m}=M_{\mathcal{T}^{*}} \phi_{m} \text { on } I \text { and } M_{\mathcal{T}} \phi_{m}=\max \left(M_{\mathcal{T}^{* *}} \phi_{m}, f\right) \text { on } X \backslash I ;
$$


hence using (1.13) and (1.14) we get

$$
\limsup _{m \rightarrow \infty} \int_{I}\left(M_{\mathcal{T}} \phi_{m}\right)^{q} d \mu \leq \tau h_{1} \omega_{q}\left(\frac{f_{1}^{q}}{h_{1}}\right) \text { if } h_{1}>0
$$

and

$$
\limsup _{m \rightarrow \infty} \int_{I}\left(M_{\mathcal{T}} \phi_{m}\right)^{q} d \mu \leq \frac{\tau f_{1}^{q}}{1-q} \text { if } h_{1}=0
$$

(where in the case $h_{1}=0$ we just used (2.2)) and

$$
\limsup _{m \rightarrow \infty} \int_{X \backslash I}\left(M_{\mathcal{T}} \phi_{m}\right)^{q} d \mu \leq\left(h-\tau h_{1}\right) \omega_{q}\left(\frac{(1-q)(1-\tau) f^{q}+q f^{q-1}\left(f-\tau f_{1}\right)}{h-\tau h_{1}}\right)
$$

if $h>\tau h_{1}$ and

$$
\limsup _{m \rightarrow \infty} \int_{X \backslash I}\left(M_{\mathcal{T}} \phi_{m}\right)^{q} d \mu \leq(1-\tau) f^{q}+\frac{q}{1-q} f^{q-1}\left(f-\tau f_{1}\right)
$$

if $h=\tau h_{1}$.

In the case $0<h_{1}<\tau h$, (5.5), (5.7), (1.16) and the concavity of $\omega_{q}$ give

$$
h \omega_{q}\left(\frac{f^{q}}{h}\right) \leq h \omega_{q}\left(\frac{\tau f_{1}^{q}+(1-q)(1-\tau) f^{q}+q f^{q-1}\left(f-\tau f_{1}\right)}{h}\right),
$$

which since $\omega_{q}$ is increasing gives $f^{q} \leq \tau f_{1}^{q}+(1-q)(1-\tau) f^{q}+q f^{q-1}\left(f-\tau f_{1}\right)$, which gives $f_{1}^{q} \geq(1-q) f^{q}+q f^{q-1} f_{1}$, which is a contradiction since $0<q<1$ and $f_{1}>f>0$.

If $h_{1}=0$, then (5.6), (5.7) and (1.16) give

$$
h \omega_{q}\left(\frac{f^{q}}{h}\right) \leq h \omega_{q}\left(\frac{(1-q)(1-\tau) f^{q}+q f^{q-1}\left(f-\tau f_{1}\right)}{h}\right)+\frac{\tau f_{1}^{q}}{1-q}
$$

and since Lemma 1 easily implies

$$
\omega_{q}(a)-\omega_{q}(b)>\frac{1}{1-q}(a-b)
$$

whenever $a>b \geq 1$, (5.10) gives $f^{q}<\tau f_{1}^{q}+(1-q)(1-\tau) f^{q}+q f^{q-1}\left(f-\tau f_{1}\right)$, which is a contradiction (since $0<\tau<1$ ) even if we had assumed $f_{1} \geq f$.

If $\tau h_{1}=h$, then (5.5), (5.8) and (1.16) give

$$
h \omega_{q}\left(\frac{f^{q}}{h}\right) \leq h \omega_{q}\left(\frac{\tau f_{1}^{q}}{h}\right)+(1-\tau) f^{q}+\frac{q}{1-q} f^{q-1}\left(f-\tau f_{1}\right),
$$

which again using (5.11) leads to a contradiction even if we had assumed $f_{1} \geq f$.

This proves (5.1) and then since

$$
f=\lim _{m \rightarrow \infty} \sum_{I \in \mathcal{T}_{(1)}} \int_{I} \phi_{m} d \mu \leq \sum_{I \in \mathcal{T}_{(1)}} \limsup _{m \rightarrow \infty} \int_{I} \phi_{m} d \mu \leq \sum_{I \in \mathcal{T}_{(1)}} f \mu(I)=f
$$

we conclude (taking subsequences) that

$$
\lim _{m \rightarrow \infty} \frac{1}{\mu(I)} \int_{I} \phi_{m} d \mu=f
$$

for every $I \in \mathcal{T}_{(1)}$. Moreover the above arguments show that for any $I \in \mathcal{T}_{(1)}$ and any subsequence $\left(\phi_{m_{j}}\right)$ such that $\lim _{j \rightarrow \infty} \frac{1}{\mu(I)} \int_{I} \phi_{m_{j}}^{q} d \mu=h_{1}$ we must have $0<\mu(I) h_{1}<h$ and then we must also have equality in (5.9), which follows from (5.5) and (5.7), which in view of the strict concavity of $\omega_{q}$ implies (with $\tau=\mu(I)$ ) 
that $\frac{f^{q}}{h_{1}}=\frac{(1-q)(1-\tau) f^{q}+q f^{q-1}(f-\tau f)}{h-\tau h_{1}}$, hence that $h_{1}=h$. Thus the first two equalities in (1.17) hold for any $I \in \mathcal{T}_{(1)}$. But since we also must have equality in (5.5), where now $f_{1}=f, h_{1}=h$, we conclude that also the third equality in (1.17) holds for any $I \in \mathcal{T}_{(1)}$. Now the proof of Proposition 1 can be easily completed by induction on the levels of the tree $\mathcal{T}$.

\section{Approximations of the Bellman functions, SCALing and KOLMOGOROV'S INEQUALITY}

Here we will derive certain estimates for the various Bellman functions in order to get some idea on what Theorem 1 says. Then we will use them to study the behavior of the dyadic maximal function on $\mathbb{R}^{n}$ and the various Bellman functions that come from (1.4). One could obtain better approximations by iterating the corresponding estimates in Lemmas 1 and 3. We first have the following.

Proposition 5. The following estimates hold:

$$
\frac{f^{q}-q f^{q-1} h^{\frac{1}{q}}}{1-q}<\mathcal{B}_{q}^{\mathcal{T}}(f, h, f, 1)<\frac{f^{q}-q(1-q)^{\frac{1}{q}-1} f^{q-1} h^{\frac{1}{q}}}{1-q}
$$

$$
\mathcal{B}_{q}^{\mathcal{T}}(f, h, L, 1)<\frac{(1-q) L^{q}+q L^{q-1} f-q(1-q)^{\frac{1}{q}-1}\left((1-q) L^{q}+q L^{q-1} f\right)^{1-\frac{1}{q}} h^{\frac{1}{q}}}{1-q}
$$

and

$$
\mathcal{B}_{q}^{\mathcal{T}}(f, h, L, 1)>\frac{(1-q) L^{q}+q L^{q-1} f-q\left((1-q) L^{q}+q L^{q-1} f\right)^{1-\frac{1}{q}} h^{\frac{1}{q}}}{1-q} .
$$

$\mathcal{B}_{q}^{\mathcal{T}}(f, h, f, k)<\frac{1}{1-q} k^{1-q}\left(f-\left(\frac{1-q}{1-q+k}\right)^{1 / q}(1-k) h^{1 / q}\right)^{q}\left(1-q\left(\frac{1-q}{1-q+k}\right)^{1 / q} k \frac{h^{1 / q}}{f}\right)$ and

$$
\mathcal{B}_{q}^{\mathcal{T}}(f, h, f, k)>\frac{1}{1-q} k^{1-q}\left(f-(1-k) h^{1 / q}\right)^{q} .
$$

(iv) The unique in $\left(0, \frac{f}{L}\right)$ solution $k_{0}=k_{0}(f, h, L)$ of the equation $H_{q}\left(\frac{L(1-k)}{f-L k}\right)=$ $\frac{f^{q}}{h} H_{q}\left(\frac{L}{f}\right)$ satisfies

$$
0<\frac{f-h^{1 / q}}{L-h^{1 / q}}<k_{0}(f, h, L)<\frac{f-(1-q)^{1 / q} h^{1 / q}}{L-(1-q)^{1 / q} h^{1 / q}}<\frac{f}{L} .
$$

Proof. (i) and (ii) follow from Corollary 1 combined with Lemma 1 (iv). For (iii) we let $\lambda=\frac{f^{q}}{h}>1$ and, using the equation satisfied by $x_{\lambda}(k)$ and (4.27), we get

$$
\mathcal{B}_{q}^{\mathcal{T}}(f, h, f, k)=h\left(\frac{x_{\lambda}(k)(1-k)}{1-k x_{\lambda}(k)}\right)^{q}-f^{q} x_{\lambda}(k)^{q}(1-k) .
$$


But (2.11) gives

$$
\left(\frac{1-k}{1-k x_{\lambda}(k)}\right)^{q}\left(1-\frac{k x_{\lambda}(k)}{(1-q) x_{\lambda}(k)+q}\right)=\lambda(1-k)=\frac{f^{q}}{h}(1-k) .
$$

Hence using (6.8) in (6.7) we get

$$
\begin{aligned}
\mathcal{B}_{q}^{\mathcal{T}}(f, h, f, k) & =f^{q} k x_{\lambda}(k)^{q} \frac{(1-k) x_{\lambda}(k)}{(1-q) x_{\lambda}(k)+q-k x_{\lambda}(k)} \\
& =f^{q} k x_{\lambda}(k)^{q}\left[1-\frac{q\left(1-k x_{\lambda}(k)\right)}{(1-q) x_{\lambda}(k)+q-k x_{\lambda}(k)}\right] .
\end{aligned}
$$

Now (6.4) follows by using (2.19) from Lemma 3 (iv) in (6.9) after observing that trivially (from $1<x_{\lambda}(k)<\frac{1}{k}$ )

$$
(1-q) x_{\lambda}(k)+q-k x_{\lambda}(k)<\max \left(1-k, \frac{(1-q)(1-k)}{k}\right)<\frac{1}{k} .
$$

To prove (6.5) we use the lower bound from (2.19) in the first equality in (6.9) after observing (now from $\frac{1}{k}\left(1-(1-k) \lambda^{-1 / q}\right)<x_{\lambda}(k)<\frac{1}{k}$ ) that

$$
\begin{aligned}
\frac{(1-q) x_{\lambda}(k)+q-k x_{\lambda}(k)}{1-k} & >\min \left(\frac{1-q}{k}\left(1-\lambda^{-1 / q}\right)+\lambda^{-1 / q}, \frac{1-q}{k}\right) \\
& >\frac{1-q}{k}\left(1-(1-k) \lambda^{-1 / q}\right) .
\end{aligned}
$$

To prove (6.6) we write (using (2.11) ) the equation for $k_{0}$ as

$$
\frac{h\left(1-k_{0}\right)^{q}}{\left(f-L k_{0}\right)^{q}}=\frac{1-k_{0}}{1-k_{0} \frac{L}{(1-q) L+q f}}
$$

and note that since $L>f$ and $0<k_{0}<\frac{f}{L}$, the right-hand side of (6.12) is between 1 and $\frac{1}{1-q}$ (one could also use Lemma 1 (iv) in (1.12) ).

Clearly the above proposition provides estimates for the full Bellman function $\mathcal{B}_{q}^{\mathcal{T}}(f, h, L, k)$ since Theorem 1 implies

$$
\mathcal{B}_{q}^{\mathcal{T}}(f, h, f, k)=\left\{\begin{array}{l}
\mathcal{B}_{q}^{\mathcal{T}}(f, h, L, 1)-L^{q}(1-k) \text { if } k \geq k_{0}(f, h, L) \\
\mathcal{B}_{q}^{\mathcal{T}}(f, h, f, k) \text { if } k<k_{0}(f, h, L) .
\end{array}\right.
$$

Now we come to the dyadic maximal operator on $\mathbb{R}^{n}$, consider a nonnegative $\phi \in L^{1}\left(\mathbb{R}^{n}\right)$, let $\|\phi\|_{1}=\int_{\mathbb{R}^{n}} \phi$ and fix a dyadic cube $Q$. Let $\mathcal{D}_{n}(Q)$ denote the tree of dyadic subcubes of $Q$ on the probability space $\left(Q, \frac{1}{|Q|}|\cdot|\right)$. Then we get the following corollary from the above proposition and Corollary 1 (where $M_{\mathcal{D}_{n}(Q)}$ denotes the local maximal operator with respect to the tree $\mathcal{D}_{n}(Q)$ and $M_{d}$ the usual dyadic maximal operator on $\mathbb{R}^{n}$ ). 
Corollary 2. We have for any $q$ with $0<q<1$,

(i)

$$
\int_{Q}\left(M_{\mathcal{D}_{n}(Q)} \phi\right)^{q}<\frac{|Q|^{1-q}}{1-q}\left[\left(\int_{Q} \phi\right)^{q}-q(1-q)^{\frac{1}{q}-1}|Q|^{1-\frac{1}{q}}\left(\int_{Q} \phi\right)^{q-1}\left(\int_{Q} \phi^{q}\right)^{1 / q}\right] .
$$

(ii) If $L=\sup _{Q^{\prime}: Q \subseteq Q^{\prime}} \operatorname{Av}_{Q^{\prime}}(\phi) \leq|Q|^{-1}\|\phi\|_{1}$, then

$$
\begin{aligned}
\int_{Q}\left(M_{d} \phi\right)^{q}<\frac{(1-q) L^{q}|Q|+q L^{q-1} \int_{Q} \phi}{1-q} & \frac{-q(1-q)^{\frac{1}{q}-1}\left(\int_{Q} \phi^{q}\right)^{1 / q}\left((1-q) L^{q}|Q|+q L^{q-1} \int_{Q} \phi\right)^{1-\frac{1}{q}}}{1-q}
\end{aligned}
$$

and

$$
\begin{aligned}
& \int_{Q}\left(M_{d} \phi\right)^{q}<\frac{|Q|^{1-q}}{1-q}\left[(1-q)\|\phi\|_{1}^{q}+q\|\phi\|_{1}^{q-1} \int_{Q} \phi\right. \\
&\left.-q(1-q)^{\frac{1}{q}-1}|Q|^{1-\frac{1}{q}}\left(\int_{Q} \phi^{q}\right)^{1 / q}\|\phi\|_{1}^{q-1}\right] .
\end{aligned}
$$

(iii) If $E \subseteq Q$ is measurable, then

$$
\int_{E}\left(M_{\mathcal{D}_{n}(Q)} \phi\right)^{q}<\frac{|E|^{1-q}}{1-q}\left[\int_{Q} \phi-\left(\frac{(1-q)|Q|}{(1-q)|Q|+|E|}\right)^{\frac{1}{q}}\left(1-\frac{|E|}{|Q|}\right)|Q|^{-\frac{1}{q}+1}\left(\int_{Q} \phi^{q}\right)^{\frac{1}{q}}\right]^{q} .
$$

(iv) With the above notation

$$
\int_{E}\left(M_{d} \phi\right)^{q}<\frac{|E|^{1-q}}{1-q}\left[\int_{Q} \phi-\left(\frac{(1-q)|Q|}{(1-q)|Q|+|E|}\right)^{1 / q}\left(1-\frac{|E|}{|Q|}\right)|Q|^{-\frac{1}{q}+1}\left(\int_{Q} \phi^{q}\right)^{1 / q}\right]^{q}
$$

when $|E| \leq k_{0}|Q|$ and

$$
\begin{aligned}
\int_{E}\left(M_{d} \phi\right)^{q}< & \frac{(1-q) L^{q}|E|+q L^{q-1} \int_{Q} \phi}{1-q} \\
& \frac{-q(1-q)^{\frac{1}{q}-1}\left(\int_{Q} \phi^{q}\right)^{1 / q}\left[(1-q) L^{q}|Q|+q L^{q-1} \int_{Q} \phi\right]^{1-\frac{1}{q}}}{1-q} 1-q
\end{aligned}
$$

when $|E|>k_{0}|Q|$, where the number $k_{0}$ satisfies

$$
\begin{aligned}
\frac{\int_{Q} \phi-|Q|^{-\frac{1}{q}+1}\left(\int_{Q} \phi^{q}\right)^{1 / q}}{L|Q|-|Q|^{-\frac{1}{q}+1}\left(\int_{Q} \phi^{q}\right)^{1 / q}}<k_{0} & <\frac{\int_{Q} \phi-(1-q)^{1 / q}|Q|^{-\frac{1}{q}+1}\left(\int_{Q} \phi^{q}\right)^{1 / q}}{L|Q|-(1-q)^{1 / q}|Q|^{-\frac{1}{q}+1}\left(\int_{Q} \phi^{q}\right)^{1 / q}} \\
& <\frac{1}{L|Q|} \int_{Q} \phi
\end{aligned}
$$

(v) Moreover when $|E| \geq \frac{1}{L} \int_{Q} \phi$ we have

$$
\int_{E}\left(M_{d} \phi\right)^{q}<\frac{L^{q}|E|}{1-q} \leq \frac{|E||Q|^{-q}\|\phi\|_{1}^{q}}{1-q} \leq \frac{|E|^{1-q}\|\phi\|_{1}^{q}}{1-q}
$$


and when $k_{0}|Q|<|E|<\frac{1}{L} \int_{Q} \phi$ we have

$$
\int_{E}\left(M_{d} \phi\right)^{q}<|Q| h \omega_{q}\left(\frac{f^{q}}{h} H_{q}\left(\frac{|Q|}{|E|}\right)\right)-f^{q}\left[\left(\frac{|Q|}{|E|}\right)^{q}-\left(\frac{|Q|}{|E|}\right)^{q-1}\right]<\frac{|E|^{1-q}}{1-q}\left(\int_{Q} \phi\right)^{q} .
$$

Proof. (i)-(iv) follow easily from the previous proposition, noting that (6.16) follows by first using the inequality $L \leq|Q|^{-1}\|\phi\|_{1}$ in (4.15) and then using Lemma 1(iv). Also from (6.19) when $|E| \geq \frac{1}{L} \int_{Q} \phi$ we easily get (6.21). When $k_{0}|Q|<$ $|E|<\frac{1}{L} \int_{Q} \phi$ we can argue as follows. We note that (with $f=\frac{1}{|Q|} \int_{Q} \phi$ and $\left.h=\frac{1}{|Q|} \int_{Q} \phi^{q}\right)$ the function $P(z)=h \omega_{q}\left(\frac{f^{q}}{h} H_{q}\left(\frac{z}{f}\right)\right)-z^{q}(1-k)$ is increasing in $z$ when $H_{q}\left(\frac{z(1-k)}{f-z k}\right) \geq \frac{f^{q}}{h} H_{q}\left(\frac{z}{f}\right)$ and $f<z<\frac{f}{k}$, the proof being similar to that of Lemma 3, using Lemma 1 and the inequality $\omega_{q}\left(\frac{f^{q}}{h} H_{q}\left(\frac{z}{f}\right)\right) \leq\left(\frac{z(1-k)}{f-z k}\right)^{q}$, to get

$$
P^{\prime}(z) \geq \frac{1}{1-q} h \frac{\frac{z(1-k)}{f-z k}}{\frac{z(1-k)}{f-z k}-1} \frac{f^{q}}{h} q(1-q) \frac{1}{f}\left(\frac{z}{f}\right)^{q-2}\left(\frac{z}{f}-1\right)-q z^{q-1}(1-k)=0,
$$

so with $k=|E| /|Q|$ we can replace $L$ by $f / k$ in the Bellman function and use Lemma 1 (ii) (a better estimate would follow from Lemma 1 (iv)) to get (6.22).

It is clear that by Proposition 5 all of the above estimates tend to be sharp when $\int_{Q} \phi$ is fixed and $\int_{Q} \phi^{q}$ tends to 0 . These thus show that Kolmogorov's inequality on $M_{\mathcal{D}_{n}(Q)} \phi$ and $M_{d}$ is sharp (when $\int_{Q} \phi^{q}$ tends to 0 ) and provide improvements, the sharp form of which is obtained by appropriately scaling the exact estimates in Theorem 1 and Corollary 1.

\section{REFERENCES}

1. D. L. Burkholder, Martingales and Fourier analysis in Banach spaces, C.I.M.E. Lectures (Varenna (Como), Italy, 1985), Lecture Notes in Mathematics 1206 (1986), 61-108. MR864712 (88c:42017)

2. D. L. Burkholder, Boundary value problems and sharp inequalities for martingale transforms, Ann. of Prob. 12 (1984), 647-702. MR744226 (86b:60080)

3. L. Grafakos, S. Montgomery-Smith. Best constants for uncentered maximal functions, Bull. London Math. Soc. 29 (1997), no.1, 60-64. MR1416408 (98b:42031)

4. A. D. Melas, The Bellman functions of dyadic-like maximal operators and related inequalities, Adv. in Math. 192 (2005), 310-340. MR2128702 (2005k:42052)

5. F. Nazarov, S. Treil, The hunt for a Bellman function: Applications to estimates for singular integral operators and to other classical problems of harmonic analysis, Algebra $i$ Analiz 8 no. 5 (1996), 32-162. MR.1428988 (99d:42026)

6. F. Nazarov, S. Treil, A. Volberg, The Bellman functions and two-weight inequalities for Haar multipliers. Journ. Amer. Math. Soc. 12 no. 4 (1999), 909-928. MR.1685781 (2000k:42009)

7. F. Nazarov, S. Treil, A. Volberg, Bellman function in stochastic optimal control and harmonic analysis (how our Bellman function got its name), Oper. Theory: Advances and Appl. 129 (2001), 393-424, Birkhäuser Verlag. MR1882704 (2003b:49024)

8. L. Slavin, V. Vasyunin, Sharp results in the integral-form John-Nirenberg inequality, submitted. 
9. L. Slavin, A. Stokolos, V. Vasyunin. Monge-Ampère equations and Bellman functions: The dyadic maximal operator. C. R. Math. Acad. Sci. Paris Sér. I, 346 (2008), 585-588. MR2412802

10. L. Slavin, A. Volberg. The explicit BF for a dyadic Chang-Wilson-Wolff theorem. The sfunction and the exponential integral. Contemp. Math. 444, Amer. Math. Soc., Providence, RI, 2007. MR2423630

11. E. M. Stein. Harmonic Analysis: Real-variable methods, orthogonality, and oscillatory integrals, Princeton Mathematical Series 43, Princeton University Press, 1993. MR 1232192 (95c:42002)

12. V. Vasyunin. The sharp constant in the reverse Holder inequality for Muckenhoupt weights. Algebra i Analiz, 15 (2003), no. 1, 73-117 MR.1979718(2004h:42017)

13. V. Vasyunin, A. Volberg. The Bellman functions for the simplest two-weight inequality: The case study. Algebra i Analiz, 18 (2006), No. 2 MR2244935 (2007k:47053)

14. V. Vasyunin, A. Volberg. Monge-Ampère equation and Bellman optimization of Carleson embedding Theorem, preprint.

15. A. Volberg, Bellman approach to some problems in Harmonic Analysis, Séminaire des Equations aux derivées partielles, Ecole Polytechnique, 2002, exposé. XX, 1-14.

16. G. Wang, Sharp maximal inequalities for conditionally symmetric martingales and Brownian motion, Proc. Amer. Math. Soc. 112 (1991), 579-586. MR.1059638(91i:60121)

Department of Mathematics, University of Athens, Panepistimiopolis 15784, Athens, Greece

E-mail address: amelas@math.uoa.gr

Department of Mathematics, University of Athens, Panepistimiopolis 15784, Athens, Greece 